\section{Patterned polymer brushes}

\section{Tao Chen, Ihsan Amin and Rainer Jordan*}

Received 23rd August 2011

DOI: $10.1039 / \mathrm{c} 2 \mathrm{cs} 15225 \mathrm{~h}$

This critical review summarizes recent developments in the fabrication of patterned polymer brushes. As top-down lithography reaches the length scale of a single macromolecule, the combination with the bottom-up synthesis of polymer brushes by surface-initiated polymerization becomes one main avenue to design new materials for nanotechnology. Recent developments in surface-initiated polymerizations are highlighted along with diverse strategies to create patterned polymer brushes on all length scales based on irradiation (photo- and interference lithography, electron-beam lithography), mechanical contact (scanning probe lithography, soft lithography, nanoimprinting lithography) and on surface forces (capillary force lithography, colloidal lithography, Langmuir-Blodgett lithography) (116 references).

\section{Introduction}

The fabrication of patterned polymer brushes on solids at the micro- and nanometre scales, with a controllable physicochemical property at a molecular level, has moved into the focus of materials science and engineering in micro- and nanotechnology. ${ }^{1}$ Because of low chain entanglement terminally attached polymer brushes are the first choice for stimulus responsive

Professur für Makromolekulare Chemie, Department Chemie, Technische Universität Dresden, Zellescher Weg 19, 01069 Dresden, Germany.E-mail:Rainer.Jordan@tu-dresden.de;

Web: http://tu-dresden.de/chemie/mc; Fax: + 49-351-46337122; Tel: + 49-351-46337676

polymer coatings for sensor and actuator developments as they react immediately to environmental changes, such as solvent quality, $\mathrm{pH}$, ionic strength, or temperature, with significant changes of the polymer layer coating. ${ }^{2,3}$ Polymer brushes are ensembles of end-tethered polymer chains with high grafting densities with respect to their radius of gyration in which the high surface crowding results in considerable stretching of the grafted chains from the substrate surface. ${ }^{4}$ They are anchored to the substrate surface by either strong physical absorption or covalent chemical attachment. ${ }^{4}$ The latter is preferred as it overcomes some of the disadvantages of physisorption, such as solvent or thermal instabilities, and

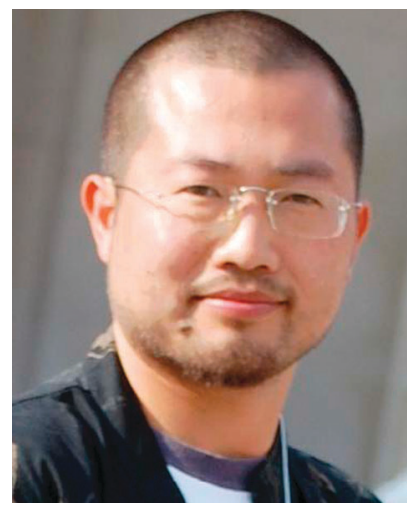

Tao Chen
Tao Chen received his $P h D$ in polymer chemistry and physics from Zhejiang University (Prof. Li Wang group) in 2006. After his postdoctoral training in Department of Chemistry at University of Warwick (Prof. Stefan A.F. Bon group), he joined Prof. Stefan Zauscher's group at Duke University as a research scientist. He is currently an Alexander von Humboldt Research Fellow hosted by Prof. Rainer Jordan at Technische Universität Dresden, Germany. He is interested in the creation and manipulation of stimulus responsive patterned bio-inspired polymeric materials and self-assembled materials system for actuation and sensing applications.

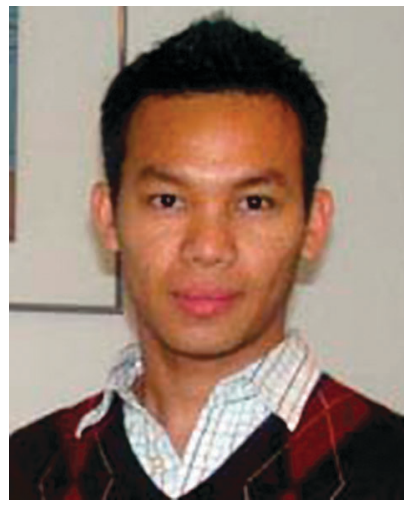

Ihsan Amin
Ihsan Amin is a postdoctoral researcher in the group of Prof. Rainer Jordan, Professur für Makromolekulare Chemie, Technische Universität Dresden, Germany. He finished his study in physics at Rijkuniversiteit Groningen, The Netherlands, and completed his doctoral studies at the Universität Bielefeld, Germany, in 2010 in the group of Prof. Armin Gölzhäuser. His research interests focus on the fabrication and application of micro- and nanopatterned stimuli responsive polymer brushes with the main interest in the development of "Polymer Carpets", freestanding polymer brushes grown by surface-initiated on crosslinked two-dimensional framework nanosheets. 
offers greater control over grafting density and yields higher packing densities.

Two fundamentally different approaches are used to realize surface-attached polymer brushes. The "grafting-to" approach involves the experimentally simple process of end-functionalized polymer chains to react with an appropriate substrate. This technique, however, has one intrinsic problem that often leads to low grafting density and film thickness due to surface-screening by already attached polymer chains. The "grafting-from" approach overcomes the shortcomings of the former as small, low molar mass monomers are directly polymerized to form the brush. Hence, surface-initiated polymerization (SIP) $)^{5-8}$ yields polymer brushes of very high grafting densities that render effectively the entire surface. Polymer brushes can be used in surface-based technologies, such as switchable sensors and actuators in micro- and nanotechnology, substrate for cellgrowth control, and for protein-resistant coatings in biological or medical fields, etc. ${ }^{2,3,8}$ Up-to-date most types of polymerization reactions from free radical polymerization to highly defined living polymerizations have been adopted to prepare polymer brushes by SIP. ${ }^{4-7}$ Because of the minute total amount of surface-bound initiation sites, living ionic polymerizations from planar substrates are experimentally very difficult to perform as they require ultraclean conditions even if a parallel sacrificial polymerization is carried out in solution. ${ }^{9,10}$ To realize structurally defined polymer brushes comprising of linear chains of low dispersity, defined end groups, block copolymer brushes by sequential monomer addition, etc., surface-initiated controlled radical polymerization is the method of choice as they are tolerant towards impurities and at the same time offer a sufficient control of the polymer architecture and composition. Hence, controlled radical surface-initiated polymerization such as atom transfer radical polymerization (SI-ATRP), nitroxide-mediated polymerization (SI-NMP) and

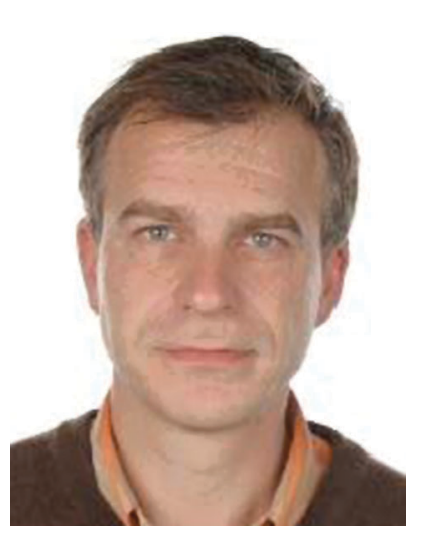

Rainer Jordan
Rainer Jordan studied chemistry at the University of Mainz (Germany) and as an IASfellow at Kyoto University (Japan) with Prof. T. Saegusa. He joined the research group of Prof. K.K. Unger in Mainz and worked as a PROCOPE-fellow at the C.N.R.S. in Paris with Prof. B. Sebille. In 1996 he obtained his doctoral degree in Chemistry with Prof. K.K. Unger (Univ. Mainz). After a postdoctoral stay with Prof. Ulman at Polytechnic University in Brooklyn, NY (USA)

he was appointed as assistant professor. He returned to Germany and joined the group of Prof. Nuyken at the Technische Universität München for habilitation. Since 2008/2009 he is full professor at the Professur für Makromolekulare Chemie at the Technische Universität Dresden, Germany. His research interests include polymer chemistry (tailored polymers), surface chemistry (self-assembled monolayers and polymer brushes), biomimetic systems (artificial cell membranes), nanoscience (composites, colloidal systems) and nanomedicine (polymer therapeutics and biomaterials). reversible addition-fragmentation chain transfer polymerization (SI-RAFT) have become the most popular routes. ${ }^{4-7}$ In this context, a recent paper by Turgman-Cohen and Genzer ${ }^{11}$ is addressing the common practice in SI-ATRP to directly correlate the molar mass and molar mass distribution of grafted polymer to the polymer grown simultaneously in solution/bulk. In contrast to the general assumption of an equal polymerization behavior, their results from Monte Carlo simulations indicate that bulk polymers grow at faster rates and possess narrower molecular weight distribution than polymers initiated from flat surfaces.

The formation of patterned polymer brushes is basically straightforward: In a top-down approach a homogeneous polymer brush is destructively patterned by selective lithography using irradiation through a mask or simply by locally confined mechanical force. However, lateral resolution will be quite limited and debris of removed material is an issue. More elegantly, a pre-patterned surface-bound initiator template can be used to amplify a two-dimensional (2D) pattern into a three-dimensional (3D) brush structure by SIP. With the requirements for such a $2 \mathrm{D}$ template system that should be of defined composition and end-function, irreversibly bound to the substrate, ultrathin to allow patterning at any length scale and of high reproducibility on a broad variety of substrate types, self-assembled monolayers (SAMs) soon became the dominant initiator system for SIP.

SAMs can be easily and reproducibly formed on almost any substrate type giving the correct choice of the anchor group and mesogen. A wide variety of head groups allow the attachment of initiator functions for all known types of SIP and it comes in handy that powerful techniques are already developed to prepare patterned SAMs. ${ }^{12}$ The two most popular and best characterized SAM types are based on silanes to modify hydroxylated surfaces such as glass or oxides and organosulfur compounds, i.e. thiols to modify coin metals. ${ }^{13-16}$ Depending on feature size and substrate material used, patterned SAMs as initiator templates for SIP can be prepared by a range of fabrication strategies including photo and interference lithography, ${ }^{17}$ electron-beam lithography (EBL), ${ }^{18}$ electron-beam chemical lithography (EBCL), ${ }^{19-21}$ scanning probe lithography (SPL), ${ }^{22,23}$ soft lithography, ${ }^{24,25}$ etc. More recently, it was demonstrated that patterned polymer brushes of defined 3D morphology can be prepared even without a surface-bound initiator by self-initiated photografting and photopolymerization (SIPGP) ${ }^{26}$ on patterned SAMs,${ }^{27,28}$ on carbon deposits (carbon templating, CT), ${ }^{29-32}$ or by the direct use of a surface chemical contrast. ${ }^{31,33,34}$ The scheme in Fig. 1 summarizes the various strategies for the preparation of patterned polymer brushes.

The goal of this review is to introduce the reader with existing lithographic techniques and their combination with surface-initiated polymerization to create patterned polymer brushes as functional surfaces. First, patterning of surfaces with irradiation ranging from UV-light to electrons is presented. Second, lithography techniques based on mechanical contact such as soft lithography, scanning probe lithography and nanoimprinting lithography are discussed and, finally, structure formation based on surface forces such as capillary force lithography, colloidal lithography and Langmuir-Blodgett lithography is summarized. 


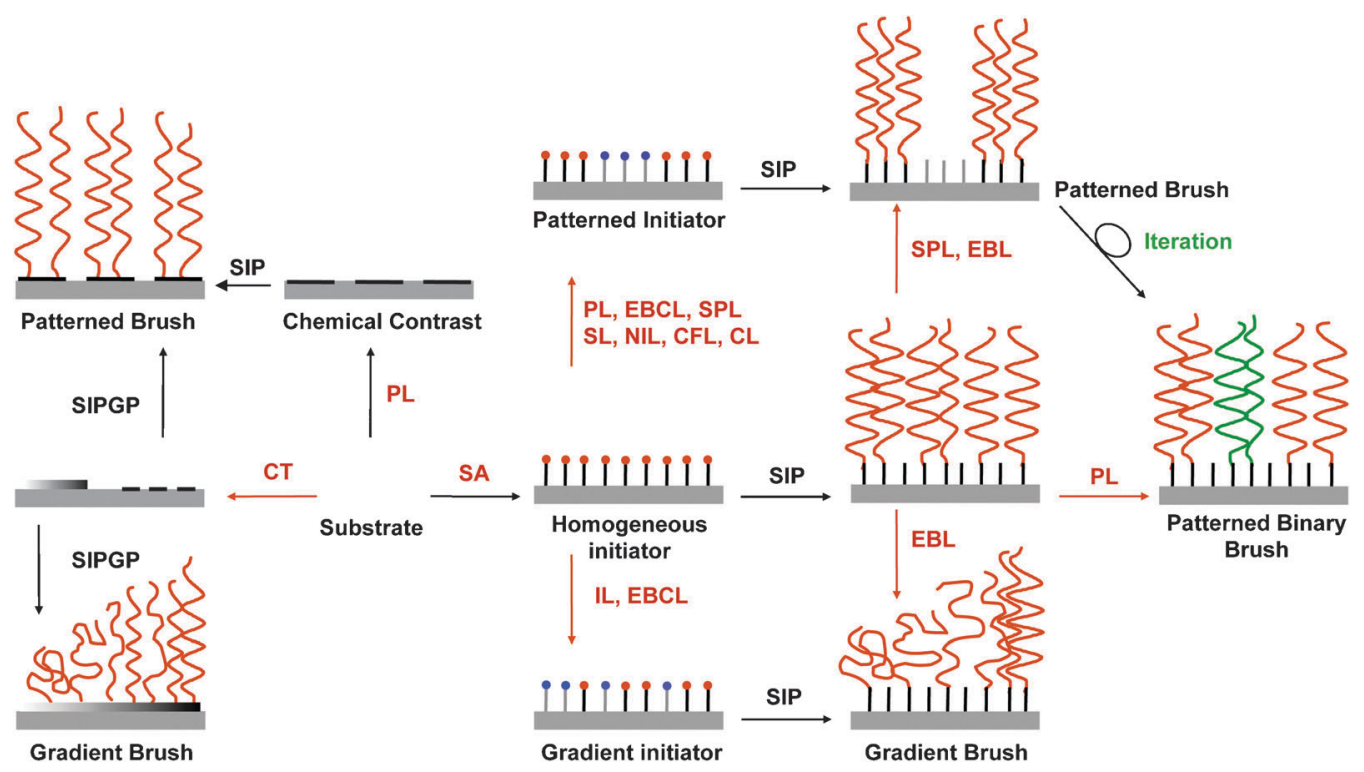

Fig. 1 Overview of various strategies for the preparation of patterned polymer brushes (Abbreviations: SIPGP: self-initiated photografting and photopolymerization; SIP: surface-initiated polymerization; CT: carbon templating; PL: photolithography; SA: self-assembly; EBCL: electron beam chemical lithography; SPL: scanning-probe lithography; SL: soft lithography; NIL: nanoimprinting lithography; CFL: capillary force lithography; CL: colloidal lithography; IL: interference lithography; EBL: electron beam lithography).

\section{Photo and interference lithography}

Lithography using irradiation of UV light, X-rays, electrons as well as ions is a widely used technique for the fabrication of micro- and nanostructured materials. As a matured technique in industry, photolithography generally involves the transfer of a mask pattern onto a substrate over large areas coated with a light sensitive polymeric photo resist and subsequent selective chemical removal of the resist. ${ }^{35}$ The remaining patterned resist is then used for a selective etching or deposition process. The resolution for photo lithography is generally determined by the diffraction limit, which is a feature size of about half the wavelength of the light used. As a consequence, especially in microchip fabrication companies pushing the limits of UV photolithography with UV sources of decreasing wavelength to fulfill Moore's law with established technology.

\subsection{UV lithography}

Rühe et $a l .{ }^{36}$ first realized the potential possibility in using photo (UV) lithography to fabricate patterned polymer brush microstructures by photo SIP in a bottom-up approach. They used a SAM of azo-functionalized alkylsilanes of AIBN-type as the photosensitive layer and irradiated the SAM through a mask. Free radical SIP (FR-SIP) occurred only at the irradiated areas. As AIBN has a quite low extinction coefficient, $\mathrm{UV}$-induced decomposition of the initiator resulted in relatively thin polymer brushes. Later, the same group introduced a more suitable asymmetric azo-functionalized SAM featuring a methylmalonodinitrile and an aryl function with higher adsorption. ${ }^{37,38}$ The thickness increase of the brush as a function of irradiation time was found to be linear with final thickness values of up to $\sim 400 \mathrm{~nm}$ after $24 \mathrm{~h}$ continuous UV irradiation.

Standard photolithography with an UV photo resist was used by Jordan and Garrido et al ${ }^{33}$ to create a chemical contrast on hydrogen-terminated diamond by plasma oxidation (Fig. 2). The patterned diamond surface with oxidized and native areas allowed selective SIP of styrene and other vinyl monomers by means of SIPGP. As SIPGP is a self-initiated polymerization and grafting reaction, it does not require a surface-bond initiator but surface groups that can be easily abstracted by a radical mechanism involving the monomer that also acts as the photo sensitizer. The high difference of the bond dissociation energies of groups in the oxidized and native diamond surface areas resulted in highly selective formation of poly(styrene) (PS) brushes only on the oxidized diamond. As no intermediate SAM is needed, the PS brushes could be subsequently converted under quite drastic reaction conditions without noticeable detachment of the polymer. Thus, various

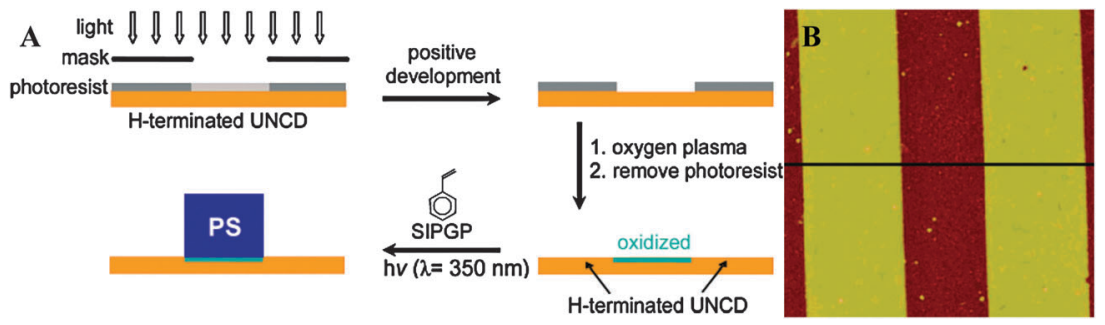

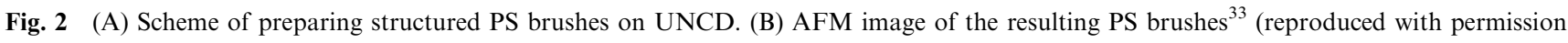
from ref. 33, copyright 2007, American Chemical Society). 

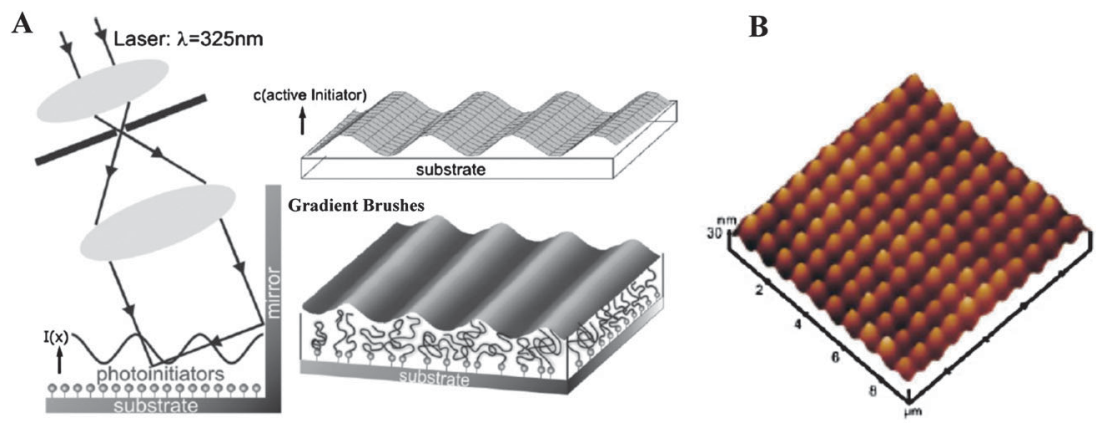

Fig. 3 (A) Outline of patterned/gradient polymer brushes by UV-interference lithography using a UV laser for IL. (B) 3D AFM height image of a PMMA brush with crossed gradient structures ${ }^{41}$ (reproduced with permission from ref. 41, copyright 2009, Wiley-VCH Verlag GmbH \& Co. KGaA).

functionalities, such as nitro, sulfonic, and aminomethyl groups could be successfully incorporated at high yields.

With UV lithography and repetitive SIP, Liu et al. ${ }^{39}$ created a binary polymer brush pattern of poly(hydroxyethyl methacrylate) PHEMA and poly(methyl methacrylate) PMMA (or poly(dimethylaminoethyl methacrylate) PDMAEMA) through a two-step SI-ATRP. First, a homogeneous polymer brush was prepared by SI-ATRP using an initiator SAM. After deactivation of the polymer chain ends by $\mathrm{NaN}_{3}$, and removal of brush regions by UV photodegradation through a mask, the native substrate areas were backfilled with the initiator SAM for a second SI-ATRP to result in a patterned binary brush covering the entire surface. The principle of a chemical patterning was further developed by Hawker et al. ${ }^{17}$ They created discrete areas of hydrophilic poly(acrylic acid) (PAA), and hydrophobic poly(tert-butyl acrylate) (PtBA) brushes derived from grafted PtBA homobrushes by photo lithography. In their approach, a solution of PS containing bis(tert-butylphenyl)iodonium triflate was spin-coated on top of a PtBA brush. UV irradiation through a mask resulted in photo acid generation confined to the irradiated areas. Diffusion of the photo acid caused local deprotection of the tert-butyl ester groups within the brush and resulted in a pattern of PtBA/PAA brushes.

\subsection{Interference lithography}

Interference lithography (IL) is a mask-free technique for patterning regular arrays of fine feature resolution for a certain wavelength without the use of complex and expensive high numerical aperture optical systems. This technique has the advantage of practically unlimited depth of focus and very large exposure fields. Generally, a linear fringe pattern with a sinusoidal intensity distribution could be formed with two or more coherent beams. In an effort to overcome some drawbacks of EBL including a limited choice of support materials that allow the formation of SAMs, and to increase patterning resolution for photo lithography, IL has thus been used to combine with other radiation source lithography, such as UV or extreme UV (EUV), to create patterned polymer brushes with nanometre resolution over large areas. ${ }^{40,41}$ This strategy was firstly exploited by Padeste et al. ${ }^{40}$ who used EUV light in a synchrotron-based interference setup to create the initiator radicals in periodic line space or dot arrays. The radicals are created in a limited depth range of about a dozens of micrometre near the surface because of the high absorption of EUV light by the substrate. In the subsequent polymerization reaction, brushes were only grafted to exposed areas.

Gradient brushes with gradual variation of e.g. the graft density, the molecular weight or the chemical composition allow a systematic variation of surface properties across the substrate and can help to improve the understanding of topography- and/or chemistry-related phenomena. ${ }^{42,43}$ Although a number of methods have been exploited to create gradient assemblies using short organic modifiers, relatively few techniques are available for generating gradient polymer brushes that rely on selective physical or chemical treatment of surfaces before or during growth of a polymer brush. ${ }^{42,44,45}$ This includes creation of density gradients for growing polymers, gradual immersion or withdrawal of a substrate from a polymerization solution, regulation of the radiation intensity during UV exposure by a shutter, etc. ${ }^{42}$ Rühe et $a l .{ }^{41}$ recently presented an elegant approach to generate gradient PMMA brushes with steep slopes at length scales down to $100 \mathrm{~nm}$ combining UV-interference lithography with SIP (Fig. 3). UV-interference is used to partially deactivate a photo initiator SAM to obtain a gradient initiator pattern. The remaining initiator is then used for surface-initiated photo polymerization and resulted in gradient polymer brushes.

\section{Electron-beam lithography (EBL)}

To realize further performance enhancement of integrated circuits one central strategy in the microelectronics industry is still to fabricate structures with smaller dimensions to cope with Moore's law. This is a driving motor for the development of lithographic technology using irradiation of decreasing wavelength. From UV, the industry moved to deep-UV and currently to EUV along with associated technological developments. EBL is currently discussed to realize further miniaturization, however, EBL involves the development of new fabrication equipment and change of process work-flow. Furthermore, it might be to slow for chip mass production unless highly parallel fabrication technologies can be developed. EBL ${ }^{46}$ was already developed in the 1960s using existing scanning electron microscope (SEM) technology and is now widely used in research and special applications. EBL can be performed using a mask or by direct writing with a focused electron-beam for substrate patterning ranging from micrometres down to a few nanometres. ${ }^{47}$ Except for maybe scanning probe lithography, the resolution of EBL has not been surpassed by any other 
lithographic methods. ${ }^{48}$ Although EBL has some drawbacks, such as high cost of the instrumentation, the need of ultra high vacuum for operation, and the inherently serial patterning, EBL is the only technique to create patterns of microscale periodicity with nanometre precision.

\subsection{Electron beam resist lithography}

EBL is almost exclusively used in resist approaches and identical as those for conventional photo resist using PMMA as the resist. The area irradiated by a focused electron beam is chemically developed to reveal the underlying substrate for selective etching and/or further modifications. The fabrication of patterned polymer brushes at the nanoscale using EBL with resists and pattern amplification by SIP was firstly reported by Zauscher et al. ${ }^{18}$ In their approach, a silicon surface is patterned with gold using "lift-off" EBL ("top-down") and the resulting pattern is then amplified by SI-ATRP ("bottom-up") to obtain $\operatorname{poly}(N$-isopropyl acrylamide) (PNIPAAM) from immobilized thiol initiator SAM (Fig. 4). Patterns with controlled feature size, shape, and periodicity could be created even over larger areas. Moreover, the surface chemistry contrast of gold patterned silicon substrates facilitates the fabrication of binary polymer brushes with high lateral resolution by using surface selective silane and thiol-based initiator SAMs.

In a similar approach Jonas et $a l .{ }^{49}$ prepared nanopatterned surfaces by EBL and silane monolayers which were later used for regio-selective growth of polymer brushes by means of SI-ATRP. ${ }^{50}$ The resulting height and width of the brush nanopatterns are analyzed by the interplay of wetting and stretching of the grafted chains at the pattern edges. Using PMMA as the photo resist material for EBL, Ober et al. ${ }^{51}$ recently reported a direct patterning strategy of a series of methacrylate polymer brushes with ester functions (poly(isobutyl methacrylate) (PIBMA), poly(neopentyl methacrylate) (PNPMA), and poly(2,2,2-trifluoroethyl methacrylate) (PTFEMA)). It is well established that patterning of positive tone methacrylate photo resists by e-beam exposure is based on the scission reactions that occur on the backbone chain. ${ }^{52}$ The reaction leads to the degradation of the polymer brush resist into smaller fragments via a radical decomposition. The increasing order of polymer sensitivity toward EBL was found to form stable radicals upon irradiation (PMMA $<$ PIBMA $<$ PHEMA $\approx$ PNPMA < PTFEMA). By destructive EBL of PMA brushes highly resolved nanostructured polymer brush patterns down to $50 \mathrm{~nm}$ lines were obtained. This method is not limited to PMA polymer brush systems but applicable to polymers that show positive tone behavior under e-beam exposure.

\subsection{Electron-beam chemical lithography (EBCL)}

3.2.1 Patterned SAM initiators. While in "lift-off" EBL, the surface materials contrast was used to realize patterned brushes, electron beam chemical lithography (EBCL) as developed by Eck et $a l .{ }^{53}$ allows the introduction of the chemical contrast within the SAM itself and thus avoids overlaying topographical features. Electron irradiation of 4-substituted aromatic SAMs results in a lateral crosslinking of the aryl mesogens along with a selective reduction of i.e. a terminal nitro to an amino group or sulfonic acid to a thiol. ${ }^{54}$ Advantageously, the lateral electron-induced conversion of 4-nitro-1,10-biphenyl-4-thiol (NBT) SAMs to crosslinked 4-amino-1,10-biphenyl-4-thiol further stabilizes the monolayer by the lateral crosslinking itself as well as by the multitude of surface attachment points of the "polymerized" SAM in the irradiated area. As the chemical conversion of the nitro to the amino group is limited to areas irradiated by electrons, the technique is referred to as EBCL. These amine terminated organic nanostructures could be used as templates for SIP using a surface-bound initiator to yield densely grafted polymer brush nanopatterns. Gölzhäuser and Jordan et al. ${ }^{19,20}$ first demonstrated the fabrication of sub-50 nm polymer brush nanopatterns by combining top-down EBCL with the bottom-up self-assembly of monolayers and SIP. SAMs of NBT were patterned by EBCL followed by diazotization and coupling of methylmalonodinitrile to result in well defined areas of crosslinked surface-bond asymmetric biphenyl/ malonodinitril azo-initiator suitable for thermal as well as photo polymerization of a broad variety of vinyl monomers. FR-SIP by thermal ${ }^{19}$ or photopolymerization ${ }^{20}$ selectively amplified the
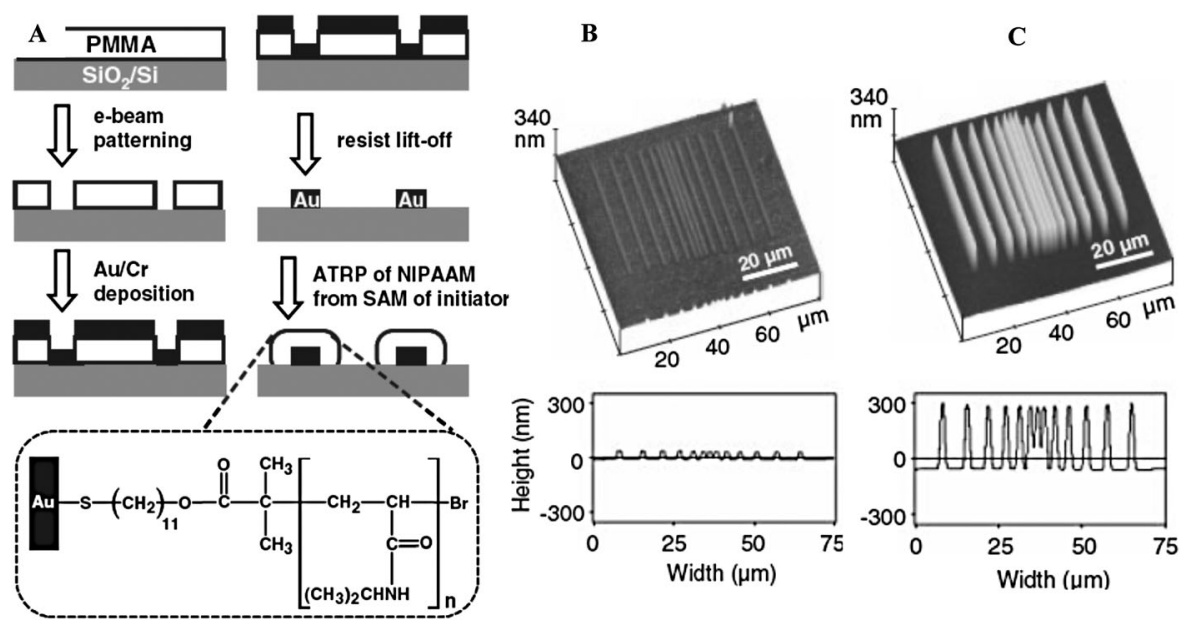

Fig. 4 (A) Stepwise fabrication of patterned PNIPAAM brushes created by EBL and SI-ATRP. (B-C) AFM scans of line patterns of gold, fabricated by EBL and subsequent PNIPAAM brush grown by SI-ATRP from immobilized thiol initiator on the Au ${ }^{18}$ (reproduced with permission from ref. 18, copyright 2004, Wiley-VCH Verlag GmbH \& Co. KGaA). 


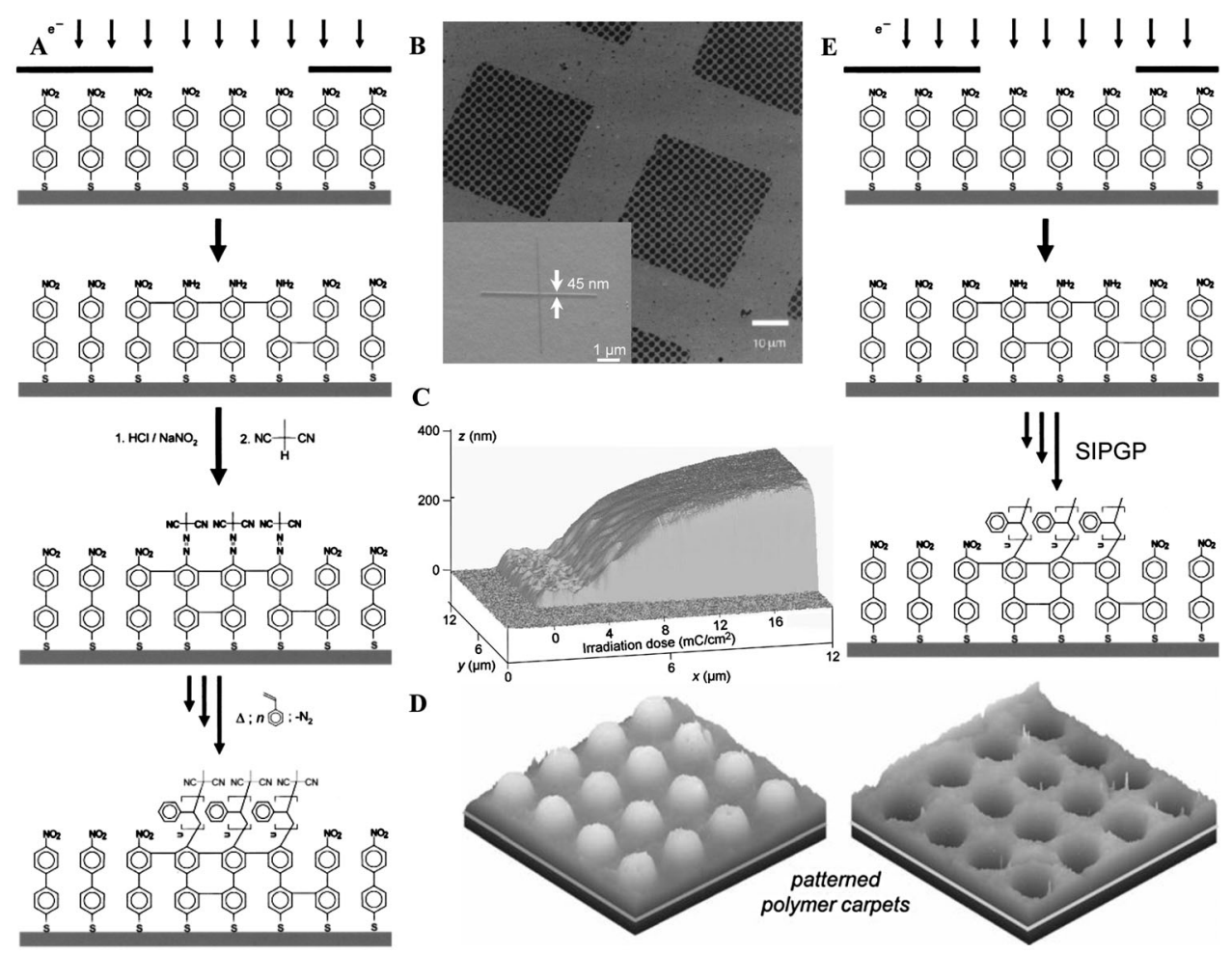

Fig. 5 (A) Scheme of preparing structured polymer brushes. (B) Electron micrographs of patterned PS brushes generated by EBCL, selective initiator coupling and SIP on the micrometre and sub-50 nanometre scale (inset) ${ }^{19,20}$ (reproduced with permission from ref. 19 and 20 , copyright 2003 and 2007, Wiley-VCH Verlag GmbH \& Co. KGaA). (C) 3D representation (AFM scan) of a continuous polymer graft gradient showing the transition from the "pancake" to the "mushroom" to the "brush" regime ${ }^{21}$ (reproduced with permission from ref. 21, copyright 2007, Wiley-VCH Verlag GmbH \& Co. KGaA). (D) 3D patterned polymer carpet ${ }^{59}$ (reproduced with permission from ref. 59, copyright 2011, Wiley-VCH Verlag $\mathrm{GmbH} \& \mathrm{Co}$ KGaA). (E) Scheme of the simplified initiator-free SIPGP.

initiator nanopatterns (Fig. 5A and B). Later, Steenackers et al. ${ }^{21}$ reported that the brush height and overall 3D morphology of the brushes can be controlled by the initial pattern size and also by the electron dose during pattern formation which defines the local initiator concentration and thus the grafting density (Fig. 5C).

3.2.2 Patterned SAMs for initiator free SIP: self-initiated photografting and photopolymerization (SIPGP). Recently, several groups $^{26,55-58}$ and we $\mathrm{e}^{27,29-34,59}$ reported that for UV-photografting of most vinyl monomers, a dedicated surface-bound initiator is not needed. Very early reports already described the phenomenon of self-initiated photografting and photopolymerization (SIPGP) on various kinds of substrates. ${ }^{60-63}$ The currently working hypothesis is based on a mechanism given by Li et al. ${ }^{64} \mathrm{UV}$ irradiation of a substrate submerged in (ideally) a liquid bulk monomer results in considerable formation of polymer brushes by a surface-initiated free-radical grafting reaction. The monomer itself acts as the photosensitizer and is able to abstract a surface moiety (e.g. a hydrogen radical) creating a free-radical site at the surface that initiates a FR-SIP. Although the SIP is a free-radical polymerization, the high concentration of monomer at the interface leads to low branching of the brushes and low degree of crosslinking. Only at longer photopolymerization times, branching and crosslinking of the grafts become apparent ${ }^{27,56}$ and surface grafting stagnates. The grafting efficiency is, among other factors (vinyl monomer, and of substrate, temperature, wavelength), ${ }^{65}$ a function of the bond dissociation energy of the surface functions. A systematic study using crosslinked SAMs bearing different terminal groups was carried out by Steenackers et al. $^{27}$ The chemical contrast of electron-beam crosslinked SAMs on otherwise native substrates was found to be sufficient for selective SIP reactions if the bond dissociation energy (BDE) of the SAM surface groups are much lower than the BDE of the groups of the substrate. The various terminal SAM functions allowed the fine-tuning of the grafting probability. The UV irradiation also desorbs non-irradiated SAM areas because of the low photostability of thiols on gold and thus the SIPGP resulted in defined polymer brush patterns. The advantage of SIPGP is that (1) no additional surface analog reaction is needed to introduce a photoinitiator for SIP, (2) very thick polymer brushes up to $1-1.5 \mu \mathrm{m}$ can be prepared, (3) layered brushes from different vinyl monomers can be prepared by consecutive SIPGP, (4) the polymer brush is directly bound to the surface via $\mathrm{C}-\mathrm{C}$ or $\mathrm{C}-\mathrm{X}$-bonds and thus very stable as compared to thiol- or silane-based SAMs, allowing polymer analog reactions under drastic conditions. ${ }^{33}$ On the other hand it must be realized that resulting brushes are characterized by some branching and dispersity values characteristic for FR-SIP. ${ }^{66}$ It is noteworthy that UV-irradiation should be at wavelength above $300 \mathrm{~nm}$ (e.g. by using Duran ${ }^{\circledR}$ or Pyrex ${ }^{\circledR}$ glassware) otherwise, significant photolytic decomposition of formed polymer brushes becomes significant. ${ }^{65}$ 


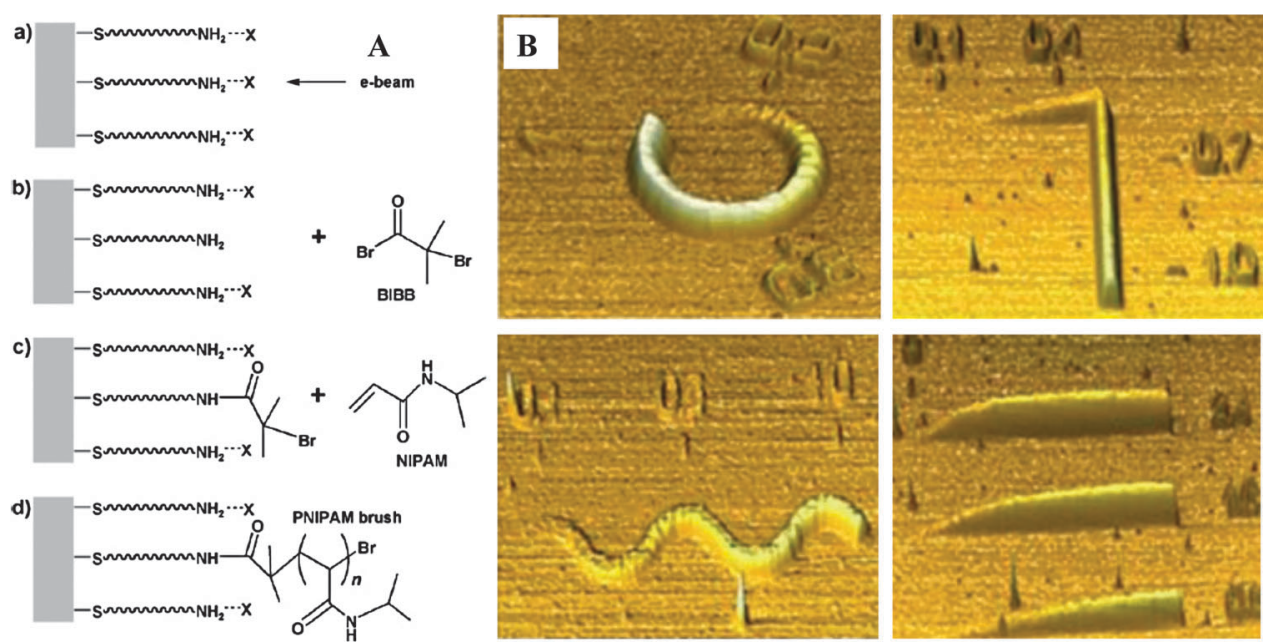

Fig. 6 (A) Fabrication scheme for a full coverage polymer brush pattern by EBCL/SI-ATRP. (B) AFM images of the resulting gradient full coverage PNIPAAM patterns $^{71}$ (reproduced with permission from ref. 71, copyright 2008, Wiley-VCH Verlag GmbH \& Co. KGaA).

Recently, the preparation of "SAMs without a substrate", so-called freestanding nanosheets prepared by electron beaminduced crosslinking of biphenyl SAMs was demonstrated. ${ }^{67}$ Nanosheets were subsequently used to prepare a "polymer brush without a substrate" termed as a polymer carpet. ${ }^{28}$ SIPGP of styrene or 4-vinyl pyridine (4VP) on chemically patterned nanosheets ${ }^{68}$ with areas of crosslinked biphenyl and $4^{\prime}$-aminobiphenyls results in patterned polymer carpets. Grafting occurs throughout the nanosheet, however, the lower BDE for an amino group results in higher local grafting densities and thus thicker brushes on these areas (Fig. 5D). Polymer carpets can be created with lateral dimensions of centimetres but thicknesses ranging from a few up to several hundreds of nanometres, depending on the SIP time. Polymer carpets, similar to substrate-bonded polymer brushes, can react spontaneously to environmental changes by swelling or collapse of the polymer layer. However, as no substrate is present, the ultrathin polymer carpet reacts immediately and with a significant morphology change (buckling). If the introduced patterning is of the same dimension as the buckling wavelength, directional-buckling occurs. The fast response, the minimal weight, the high aspect ratio, the anisotropical buckling of patterned (stimuli-responsive) polymer carpets on a nanometre thin 2D lattice render this system most suitable for sensing and next-generation nano- or microchemomechanical systems (MCMS) ${ }^{69}$ Recently, the $1 \mathrm{~nm}$ thick nanosheet could be replaced by atomically thin single layer graphene for the production of patterned polymer carpets on the 1 atom thick graphene layer. Patterned polymer carpets on graphene was realized either by SIPGP of styrene using a mask during UV irradiation or by $\mathrm{CT}$ with various other vinyl monomers. ${ }^{32}$

To meet the demand of commercially available SAMs as resists, Zharnikov and coworkers developed EBCL on aliphatic SAMs of dodecanethiol (DDT) as resists. ${ }^{70}$ After electron irradiation, the patterns were displaced with 11-aminoundecanethiol (AUDT). This resulted in amino-terminated AUDT templates in a background of DDT. An ATRP initiator was then attached to the patterned AUDT templates for the SIP of NIPAAM.
Furthermore, the same group showed the fabrication of full-coverage but topographically patterned brushes by e-beam activation of the SAM. Although the mechanism of activation and chemical identity of initiating species is not clear, they demonstrated the preparation of well-defined brush structures of PNIPAAM (Fig. 6). ${ }^{71}$

EBCL has thus opened a new window of opportunities for the creation of polymer brush nanostructures with high resolution and fidelity. EBCL offers the capability to build at both the micro and nanoscale while imparting great control over the grafting density and height of the polymer brushes.

\subsection{Electron beam induced carbon deposition}

Although surface-bounded initiator SAMs are a common choice to act as 2D templates for selective grafting of polymer brushes, there are several drawbacks associated to the SAM system such as their limited thermal and chemical stability. For example, silane monolayers are prone to hydrolysis and their poor long term stability in saline solutions at $37{ }^{\circ} \mathrm{C}$ renders them inappropriate for long-term biomedical applications. Thiol-based SAMs desorb at elevated temperatures or longer UV irradiation. Furthermore, the multi-step procedure of SAM formation, structuring, and functionalization to introduce a suitable initiator template for the SIP is probably too demanding for technically relevant applications. Since, in SIPGP, the monomer itself acts as a photosensitizer leading to surface radicals by abstraction, virtually any organic layer that is locally deposited can act as a $2 \mathrm{D}$ template for regio-selective grafting. Taking advantage of the high resolution of e-beam writing and the fact that electron irradiation of any surface (except gold) leads to deposition of carbon with a composition close to $\mathrm{C}_{9} \mathrm{H}_{2} \mathrm{O}$ with $90 \% \mathrm{sp}^{2}$ and $10 \% \mathrm{sp}^{3}$ carbon. Steenackers et al. $^{29}$ developed a resist-free, mask-free and initiator-free technique to prepare highly stable patterned polymer brush surfaces by carbon templating (CT) (Fig. 7A) ${ }^{72}$ The residual hydrocarbons present in the vacuum chamber of an electron microscope are the source for the carbonaceous deposits that are highly cross-linked, thermally and chemically stable, and contain diverse functionalities including $\mathrm{C}-\mathrm{H}, \mathrm{C}-\mathrm{O}$, and $\mathrm{OH}$ groups, 

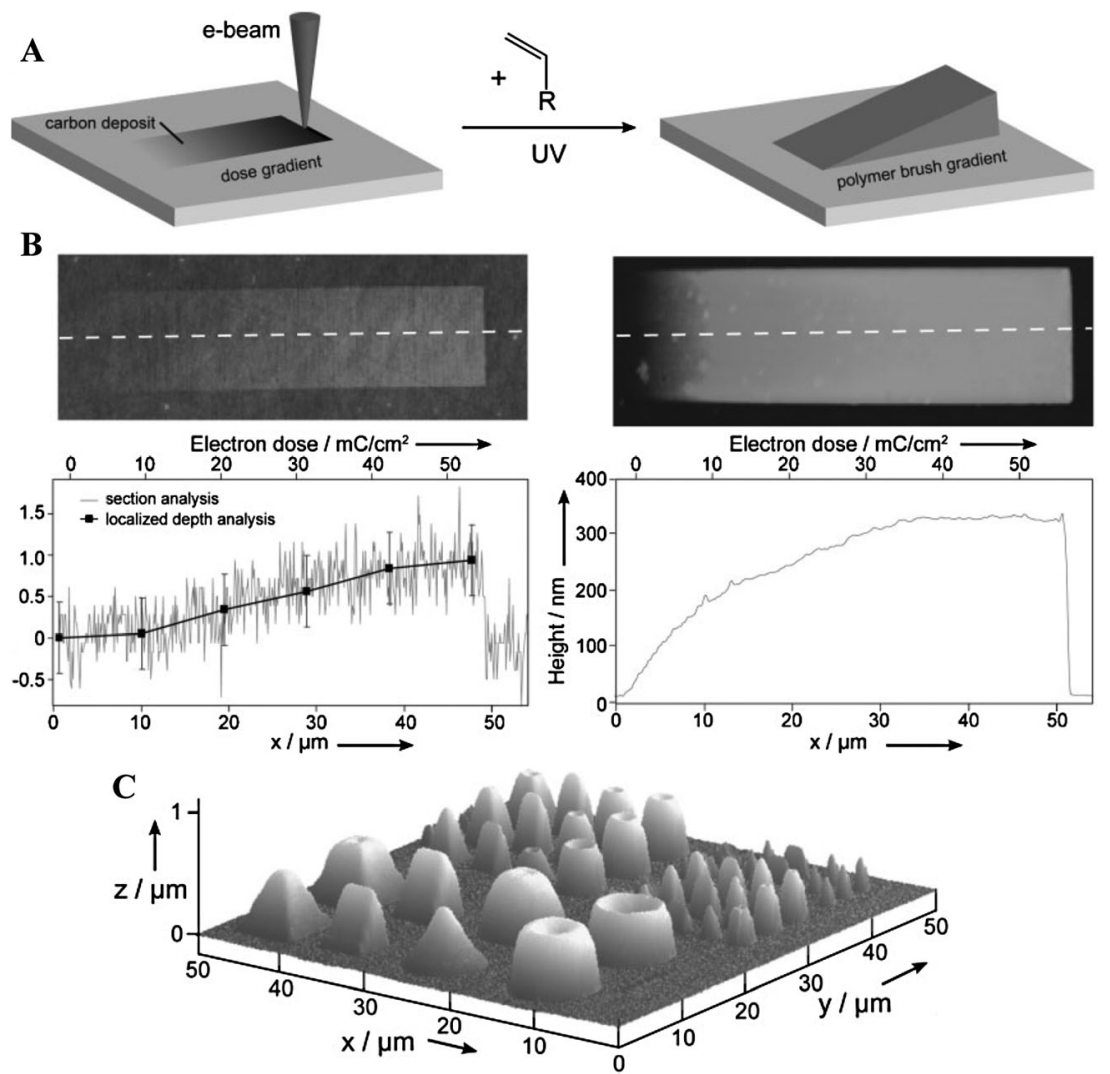

Fig. 7 (A) Scheme of carbon templating (CT) to create patterned brushes of controlled morphology directly on native substrates. (B) AFM analysis of a gradient carbon deposit, and the resulting gradient PS brushes. (C) AFM scan of PS brush objects of different sizes and shapes on a native gallium arsenide substrate ${ }^{29}$ (reproduced with permission from ref. 29, copyright 2009, Wiley-VCH Verlag GmbH \& Co. KGaA).

suitable for the SIPGP. A carbon template gradient was prepared on a native silicon/silicon dioxide substrate by EBCD with direct e-beam writing. In agreement with earlier reports, the resulting thickness of the carbon deposits is a function of the locally applied electron dose (Fig. 7B). The ultrathin carbon deposit ( 0 to $1 \mathrm{~nm}$ ) pattern could be directly amplified via SIPGP into a three-dimensional polystyrene brush gradient (0 to $300 \mathrm{~nm}$ ). AFM measurements and fluorescence microscopy revealed that the photografting occurs selectively on the carbon deposits and the height of resulting polymer brush gradient of the amount of deposited carbonaceous deposits was controlled by the locally applied dose. With CT, arrays of complex 2D brush structures such as gradients, cones, pyramids, and cups of lateral dimensions from the micrometre to the sub- $100 \mathrm{~nm}$ scale can be realized (Fig. 7C). Moreover, as $\mathrm{CT}$ is applicable to almost all inorganic substrates, patterned polymer brushes can be created on almost any surface regardless of the surface chemistry of the substrate. This was demonstrated for all kinds of substrates including metal oxides, common semiconductors ${ }^{29}$ as well as otherwise inert carbon based materials such as diamond, ${ }^{34} \mathrm{SiC}^{31}$ and very recently graphene. ${ }^{32}$ Taking advantage of the high resolution of direct e-beam writing used for $\mathrm{CT}$ polymer brushes with a footprint dimension as small as $5 \mathrm{~nm}$ was recently demonstrated..$^{30}$ As the brush is grafted directly onto the carbon deposits, consecutive polymer analog reactions under quite drastic conditions are possible. In the same account, this was used to create protein density gradients by various polymer analog conversions without noticeable loss of grafted polymer chains. With respect to the possible patterning resolution and selectivity of grafting, CT is a good alternative to scanning probe-based lithography using SAMs such as the dip-pen nanolithography (DPN) developed by Mirkin and coworkers. ${ }^{73}$

\section{Scanning probe lithography (SPL)}

Scanning probe lithography (SPL) uses a scanning tunneling microscope (STM) or the atomic force microscope (AFM) to fabricate nanometre-scale features. ${ }^{74-77}$ Similar to CT, SPL has the advantages over other available nanofabrication techniques because of its simplicity and dual capability to image and manipulate nanostructures on surfaces. Moreover, SPL can be carried out in ambient conditions of temperature and pressure and can be performed in different solvent or buffer environments with a minimum of sample preparation. However, since SPL is as CT a linear writing process its throughput is limited. Nevertheless, SPL provides a broad arsenal of approaches for pattern generation, based on various chemical, physical and electrical modifications of surfaces, including decomposition of SAMs,${ }^{78}$ mechanical scratching, ${ }^{79}$ and electrochemical anodization of silicon surfaces. ${ }^{80}$

SPL based patterning strategies were subsequently employed in the fabrication of patterned polymer brushes. The majority of patterning efforts using SPL has been directed towards fabricating templates for subsequent modification with initiators 
and amplification to polymer brushes. A unique feature of SPL based techniques is the intimacy of contact between tip and substrate surface which provides the ability to deposit initiator directly or to trigger polymerization within a small reaction volume in the meniscus around the scanning probe tip. ${ }^{81}$

\subsection{Dip-pen nanolithography}

Dip-pen nanolithography (DPN) developed by Mirkin et al. ${ }^{73}$ is a versatile technique to generate nanopatterns on surfaces. Originally, this method uses an AFM tip as a "pen", with molecules as an "ink", to write a pattern on a solid substrate as a "paper". The molecules on the AFM tip are transported to the substrate by a diffusional mechanism. DPN is comparable to microcontact printing ( $\mu \mathrm{CP})$, however, if DPN is analogue to writing with a pen, $\mu \mathrm{CP}$ is a printing press. DPN allows a better resolution $(\sim 15 \mathrm{~nm})$ as compared to $\mu \mathrm{CP}$ and a minimum feature size below $50 \mathrm{~nm}$ can be obtained. DPN can generate thiol SAM patterns in a dry nitrogen environment, since a water meniscus is always present, even at $0 \%$ relative humidity. ${ }^{82}$ During the DPN process, the surface water meniscus acts as a blocking layer for hydrophobic molecules such as 1-octadecanethiol (ODT). In the case of hydrophilic molecules, such as 16-mercaptohexadecanoic acid (MHA), it allows thiol transport to the gold surface. ${ }^{82}$ Liu et al. ${ }^{83}$ combined DPN and ROMP to fabricate polymer brush arrays on the nanometre length scale with great control over feature size, periodicity and shape (Fig. 8). In this approach, norbornenylthiol molecules were first patterned onto a gold substrate which was then passivated by a DDT solution and subsequently reacted with Grubbs' first generation catalyst and norbenyl monomers, yielding a polynorbornene brush array with $78 \mathrm{~nm}$ feature size.

Zapotoczny et al. ${ }^{84}$ reported another approach where surfacebound gold nanostructures were fabricated by DPN and subsequently used as templates for the photo initiated polymerization of methylmethacrylate, yielding a lateral resolution of only $20 \mathrm{~nm}$. Maynor et al. ${ }^{81}$ used a electrochemical dip-pen nanolithography (E-DPN) to polymerize 3,4-ethylenedioxythiophene (EDOT). This approach yielded conductive pEDOT nanostructures with sub-100 $\mathrm{nm}$ dimensions on semiconducting and insulating surfaces.

\subsection{Nanoshaving and nanografting}

While imaging with the AFM in contact mode, the force between the tip and the sample is a major concern as it may cause significant damage to the sample. Interestingly, this mechanical force can be harnessed in a controlled way to specifically create nanostructures. ${ }^{85}$ Compared with other lithographic techniques, nanoshaving is a relatively simple and basic patterning method, where a soft SAM resist on a gold substrate is removed to create patterns by the cantilever tip. The large contact pressure during patterning causes the displacement of molecules. In nanoshaving, structures are formed either in air or under a solvent and the sample is then immersed in a different thiol solution. The use of a solvent generally produces better resolution as it minimizes the readsorption of the displaced species. In nanografting, ${ }^{86}$ the same lithographic mechanism as in nanoshaving is applied, but the cantilever and SAM are immersed in a thiol solution, and new thiol self-assembles on the scratched area.

Liu et al. ${ }^{79}$ determined important parameters for nanoshaving and nanografting such as the scanning force and speed, the concentration of alkanethiol solution, and the sharpness of the AFM tip. Their work suggests that the sharpness of the tip is a critical parameter, while the concentration of the thiol solution and the scanning speed are less important for patterning. Using sharp tips, nanoshaving and nanografting can routinely generate sub-10 $\mathrm{nm}$ patterns. The resulting patterns can be used as templates for SIP. For example, PNIPAAM brush nanopatterns were prepared by Zauscher et al. ${ }^{22}$ via nanoshaving and backfilling with an ATRP initiator SAM (Fig. 9). They were able to fabricate polymer brush nanopatterns with an aspect ratio (height/width) of about $1 / 10$ in the swollen state. Further improvements to reduce feature dimensions, in the nanoshaving process by the use of sharpened probe tips, closed-loop position control of the XY-scanner, and careful control of the shaving conditions of speed, time and applied force can be expected.

Analog to the CT approach $^{29}$ and the 3D-morphology control of nanopatterned polymer brushes by EBL, ${ }^{20,21}$ Zheng et $a{ }^{87}$ employed DPN for the regio-selective deposition of ATRP initiators. The amplification of the 2D-initiator pattern

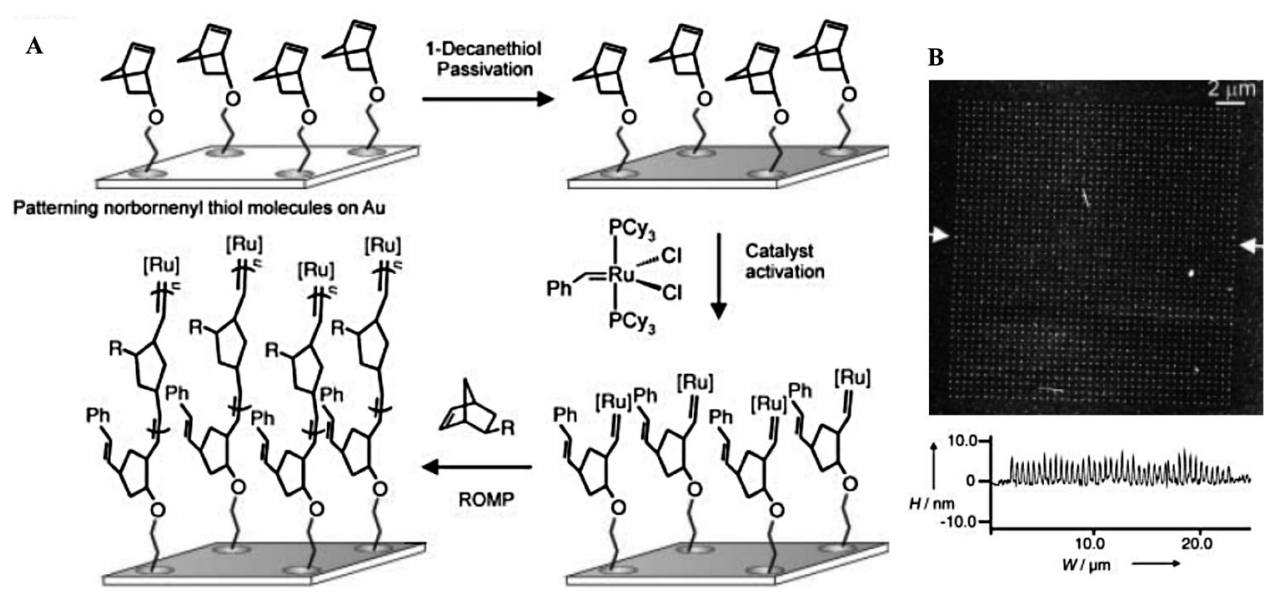

Fig. 8 (A) Preparation of poly(norbornene) brush by DPN and ROMP. (B) AFM image of polymer brush lines and dot arrays ${ }^{83}$ (reproduced with permission from ref. 83, copyright 2003, Wiley-VCH Verlag GmbH \& Co. KGaA). 

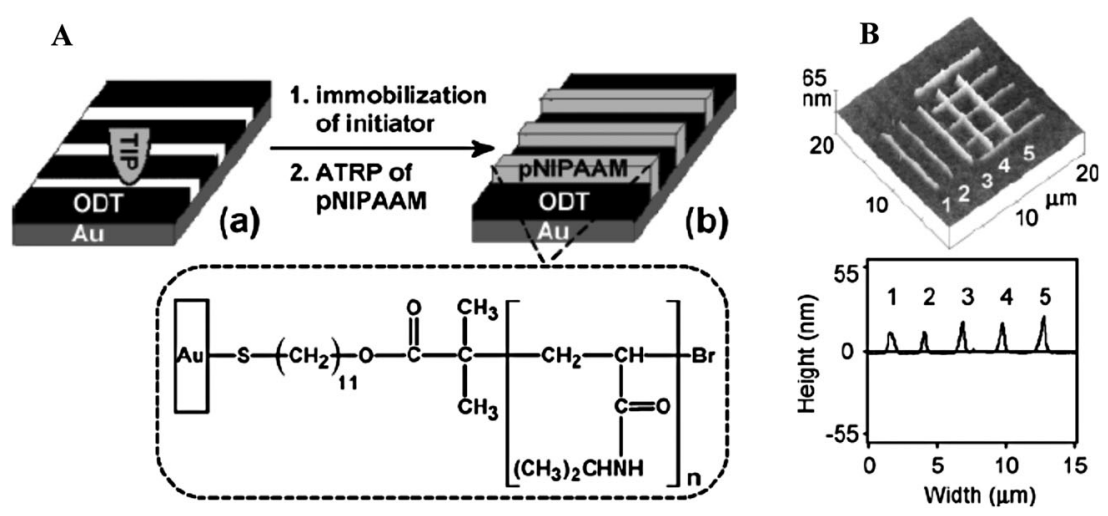

Fig. 9 (A) Preparation of patterned PNIPAAM brushes by combining nanoshaving with SI-ATRP. (B) AFM height images and corresponding typical height profiles of a PNIPAAM brush line nanopattern ${ }^{22}$ (reproduced with permission from ref. 22, copyright 2004, American Chemical Society).

with controlled spacing of initiator molecules resulted in a 3D-brush nanostructure of distinct morphology. The potential of DPN was fully utilized to obtain highly complex 3D patterns (e.g. image of the Mona Lisa). Chi and Studer et al. ${ }^{88}$ recently developed the nanoshaving strategy to mechanical nanoscratching by AFM lithography to structure a polymer brush coated silica substrate. They demonstrated substantial differences between nanoscratched spin-coated polymer layers and polymer brushes during high-loading-force AFM lithography. Polymer brushes with a thickness of $20-30 \mathrm{~nm}$ can be reproducibly structured and scratched down to the silicon substrate even with single scan lines without dragging of material in the vicinity. Equidistance line structures with $100 \mathrm{~nm}$ resolution were achieved in such a simple destructive process.

\subsection{Anodization lithography}

AFM anodization lithography is an electrochemical lithography process in which a voltage bias applied to an AFM tip, establishes a strong, localized electric field between the tip and substrate surface, and causes oxide growth on semiconducting silicon oxide substrates. The mechanism of AFM anodization lithography has been reported first by Gordon et al. ${ }^{89}$ who suggested that the electric potential produces oxyanions, such as $\mathrm{O}^{-}$and $\mathrm{OH}^{-}$, electrochemically at the air and oxide interface and that oxidation thus also promotes hydrolysis of $\mathrm{Si}-\mathrm{O}$ bonds. The factors affecting patterning are the applied electric potential between tip and surface, the relative humidity, the electronic state of tip and surface materials, and the patterning speed.

\begin{abstract}
A
\end{abstract}
(a)
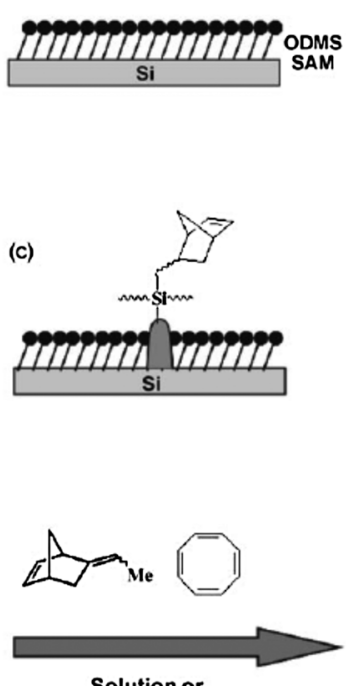

Solution or Vapor phase (b)
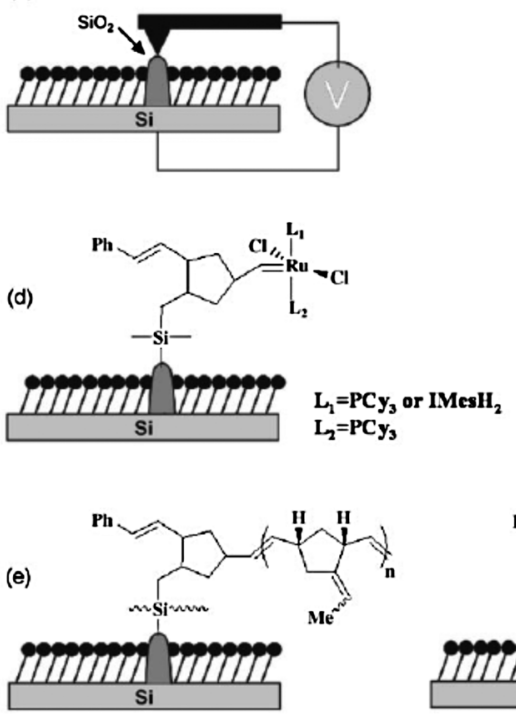

polyENB

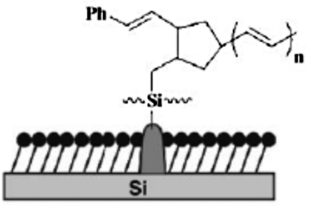

polycot
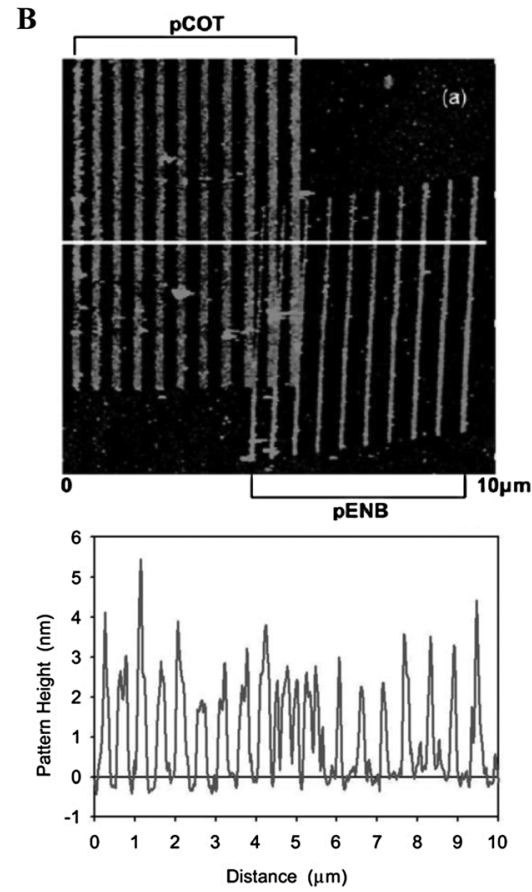

Fig. 10 (A) Stepwise fabrication schemes of nanopatterned pENB and pCOT by using anodization lithography and ROMP. (B) Height images, and the cross-sectional profile, of binary polymer brushes on the same substrate fabricated by a step and repeat process shown in $(\mathrm{A})^{90}($ reproduced with permission from ref. 90, copyright 2006, Wiley-VCH Verlag GmbH \& Co. KGaA). 
Zauscher and Caster et al. ${ }^{90}$ combined anodization lithography with ROMP to fabricate spatially well-defined pCOT (poly-cyclooctatetraene) and pENB (poly-5-thylidene-2-norbornene) brushes with a feature size of about $200 \mathrm{~nm}$ (Fig. 10). New anodic oxide patterns, next to already existing polycot nanopatterns, can be generated and then amplified the new pattern by ROMP of ENB, using Grubbs' catalyst. Although ROMP is a living polymerization, the relatively stable, chain-terminated ruthenium polycot likely did not survive the extensive cleaning procedure that followed the first step; this allowed a height control of the newly created pENB pattern to yield a similar value as that of the pCOT pattern.

\section{Soft lithography}

Soft lithography represents a non-photolithographic strategy based on self-assembly and replica molding for micro- and nanofabrication. ${ }^{13}$ It was developed in the early 1990 s by the Whitesides group and allows an easy way to fabricate micropatterned SAMs on gold by using an elastomeric stamp. ${ }^{91}$ It provides a convenient, effective, and low-cost method for the formation and manufacturing of micro- and nanostructures even over large areas, and has since been used by countless research groups. In soft lithography, feature sizes ranging from $30 \mathrm{~nm}$ to $100 \mu \mathrm{m}$ can be produced. ${ }^{13}$ Among several main techniques developed in soft lithography, microcontact printing $(\mu \mathrm{CP})$ is the original and most attractive soft lithographic tool that uses the relief pattern on the surface of a stamp to form patterns of SAMs on the surfaces of substrates upon mechanical contact. Once the stamp is available, multiple copies of the pattern can be easily produced with good reproducibility.

Crucial for $\mu \mathrm{CP}$ is the conformal contact of the stamp with the substrate surface. An elastomeric stamp, typically made from poly(dimethylsiloxane) (PDMS), is usually prepared by replica molding in which the liquid prepolymer of an elastomer is casted onto a master fabricated by e.g. conventional photo lithography. The chemical and mechanical properties of the stamp are two of the important elements in $\mu \mathrm{CP}$. Native PDMS stamps are hydrophobic and work well for e.g. aliphatic thiol "inks". To deposit hydrophilic molecules, the PDMS stamp surface has to be rendered hydrophilic via plasma oxidation, UV-ozone treatment or chemical modification. ${ }^{92}$ The feature size and stamping fidelity are determined by the design and the mechanical properties of the polymeric stamp, the diffusivity of the ink, and the contact time and applied pressure during stamping and things get increasingly complicated as pattern resolution goes up. However, to create defined patterns on the micrometre scale at extremely low costs and a minimum of technology involved (can be performed by hand) $\mu \mathrm{CP}$ is the easiest technique to obtain patterns of very high reproducibility and quality. $\mu \mathrm{CP}$ is thus also the simplest method to create micrometre patterns of patterned 2D-initiators of silanes on $\mathrm{Si} / \mathrm{SiO}_{2}$ wafers or glass and thiols on gold for structure and chemical contrast amplification by SIP.

\subsection{Micro contact printing $(\mu \mathrm{CP})$}

Very soon, $\mu \mathrm{CP}$ was used to fabricate patterning polymer brushes and became one of the most frequently used methods to fabricate patterned polymer brushes with a feature size at the microscale. While early examples involved printing of inert SAMs (e.g. octadecyltrichlorosilane, OTS) pattern to direct the backfilling of the interspaces with an initiator, followed by a SIP,${ }^{24}$ more recently, the initiator SAM is directly printed for subsequent SIP. ${ }^{25}$ Abbott, Hedrick and Hawker et al. ${ }^{24}$ first used $\mu \mathrm{CP}$ as a lithographic tool for the fabrication of patterned polymer brushes. They initially printed a non-reactive SAM of $\mathrm{CH}_{3}-\left(\mathrm{CH}_{2}\right)_{15} \mathrm{SH}$ onto a gold surface with a selective backfill of a second thiol of $\mathrm{HO}\left(\mathrm{CH}_{2} \mathrm{CH}_{2} \mathrm{O}\right)_{2}\left(\mathrm{CH}_{2}\right)_{11} \mathrm{SH}$ onto the bare gold regions. This results in the formation of a surface hydroxyl pattern that could be amplified regio-selective surface-initiated ring opening polymerization of $\varepsilon$-caprolactone.

An interesting approach to fabricate patterned multi-component polymer brushes of high complexity was demonstrated by Huck et al. (Fig. 11). ${ }^{25}$ First, a patterned initiator SAM was prepared by $\mu \mathrm{CP}$ and used for SI-ATRP. After deactivation of the dormant chain end a new initiator pattern can be printed and used for SI-ATRP with another type of monomer to result in a binary brush. Repetition of $\mu \mathrm{CP}$, SI-ATRP and deactivation up to a quaternary brush was demonstrated. This repetitive approach yields patterned polymer brushes of high chemical contrast. Interestingly, the repetition of $\mu \mathrm{CP}$ and brush growth also offers the possibility to create nanostructured brushes by using the gap caused by incomplete contact printing because of brush screening.

\subsection{Extended micro contact printing}

To date, the development of $\mu \mathrm{CP}$ has exceeded the original aim of replicating PDMS stamp patterns. Some new patterned micro/nanostructures that do not exist on the original stamp could be achieved by extending $\mu \mathrm{CP}$ of a physical deformation via lateral compression or solvent swelling to a stamp, ${ }^{93}$ alternatively, of a treatment by plasma or UV-ozone to a single stamp feature surface along with a chemical functionality change of the stamp surface. ${ }^{94}$ Extended $\mu \mathrm{CP}$ open a window to fabricate more complex patterns with the same PDMS stamp but under various printing conditions. There are considerable demands to pattern complex polymer brush microstructures because the properties of materials are highly dependent on the complexity of structures in practical applications. These complex polymer brushes have been structured via several elegant patterning strategies, e.g. EBCL, ${ }^{19,20,70,95}$ or EBCD. ${ }^{29}$ However, they are not accessible to a number of researchers because of expensive and complex instruments used. Thereafter, exploiting more simple strategies motivates several current endeavors to fabricate complex polymer brush morphologies by $\mu \mathrm{CP}$ via adjusting simply printing conditions.

Huck et al. ${ }^{96}$ prepared hierarchically well-defined structured polymer brush microstructures via multiple step $\mu \mathrm{CP}$ with inks containing different ratios of inert along with initiatorfunctionalized thiols. Zauscher et $a .^{97}$ presented a simple strategy to fabricate egg-cup shaped polymer brush microstructures. They use the microphase separation of binary thiol mixtures during the printing process ${ }^{98}$ to yield initiator gradient patterns that can be amplified into egg-cup shaped polymer brush microstructures by SI-ATRP. Chen et al. ${ }^{99}$ recently reported on the fabrication of ring shaped polymer brush structures using non-wetting conditions of an hydrophilic oxidized PDMS stamp to print hydrophobic initiator-thiols. During printing the thiols 


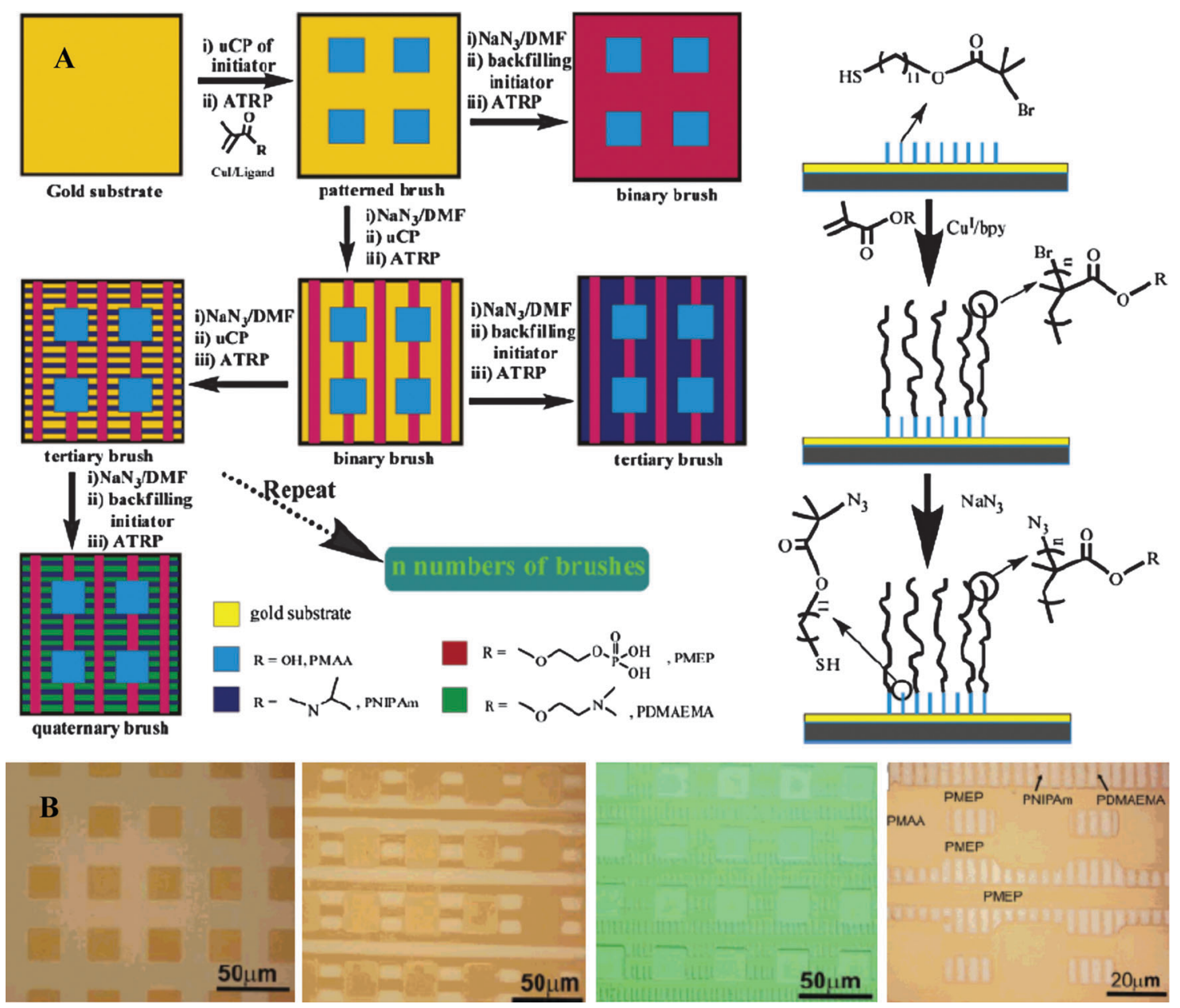

Fig. 11 (A) Outline procedure for grafting multiple patterned polymer brushes by $\mu C P$, and ATRP. (B) Evolution of optical microscopic images of patterned polymer brushes following the fabrication process shown in (A) ${ }^{25}$ (reproduced with permission from ref. 25, copyright 2006, American Chemical Society).

are only transferred along the rims of the stamp contacts. The 2D circular SAM patterns could be selectively amplified by SI-ATRP into the corresponding brush patterns. Even more complex structures can be prepared by moving the stamp during the $\mu \mathrm{CP}$ printing process. This "dynamic $\mu \mathrm{CP}$ "100 is a low-tech approach to prepare new complex (hierarchical and gradient) patterns of SAM mixtures that can readily be amplified by e.g. SI-ATRP into 3D brush structures.

In addition to direct printing using a PDMS stamp to transfer the ink pattern, it also could be extended to use relief structures on the stamp surface to conformal contact with substrate surface for forming a microchannel. Chow and Chilkoti ${ }^{101}$ reported their effort to use such a microchannel to structure biopolymer brushes by a surface-initiated enzymatic polymerization (SIEP) using terminal deoxynucleotidyl transferase (TdTase). In this approach, a plasma oxidized stamp, with a hydrophilic surface, was placed on a gold substrate and sealed by the application of gentle pressure. Next, an oligonucleotide-thiol (DNA-thiol) was pipetted into one end of the microchannels and allowed to spread through the microchannels via capillary force. The micropatterned surface was then washed with phosphate buffered saline (PBS) to remove nonspecifically bound DNA-thiol, followed by drying the surface with a stream of nitrogen. After the back-filling with nonreactive thiol, a biological polynucleotide brush was grown by SIEP by incubating the patterned oligonucleotide SAM substrate into a mononucleotide solution.

\section{Other lithographic approaches}

Although the main pattern strategies for structured polymer brushes have been stated above, there are still many other endeavors to apply the existing lithographic tools to obtain patterned polymer brushes micro/nano structures.

\subsection{Nanoimprint lithography (NIL)}

Nanoimprint lithography (NIL), initially invented and developed by Chou et al. ${ }^{102}$ in $1990 \mathrm{~s}$, is a major breakthrough in nanopatterning because it has the advantage over other currently conventional lithography in producing sub-10 $\mathrm{nm}$ feature size over a large area with a high throughput and low cost. This is the key issue why NIL has attracted wide attention within only a few years after its inception. Nevertheless, the principle of NIL is quite simple. NIL patterns a resist by deforming the polymer resist shape through embossing, with a hard mold containing nanoscale features on its surface, under controlled temperature 


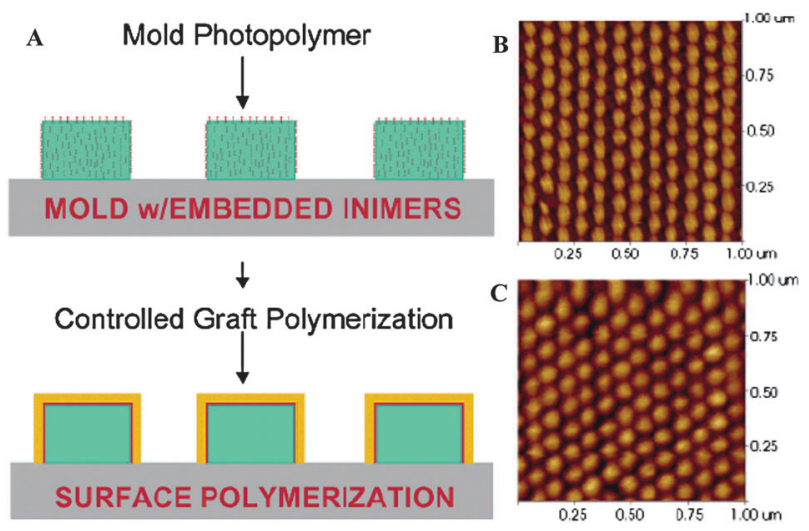

Fig. 12 (A) Graphical scheme of patterning polymer brushes by NIL/ ATRP. AFM images of contact-molded pillars of $60 \mathrm{~nm}$ before polymer brush growth (B), $75 \mathrm{~nm}$ pillars after brush growth $(\mathrm{C})^{103}$ (reproduced with permission from ref. 103, copyright 2003, American Chemical Society).

and pressure, rather than by altering resist chemical structures through radiation. After imprinting the resist to create a thickness contrast in the polymer resist, an anisotropic etching is used to remove the residue resist in the compressed area to expose the underneath substrate. For this fast developing technique, there are still some new challenges of handling complex patterns, and great demand for new material systems with properties more suitable for the imprint application.

This fabricating strategy was then carried out to pattern polymer brush nanostructures by Carter and Hawker et al., ${ }^{103}$ who employed a top-down nanocontact molding process, followed by the controlled growth of polymer brushes from these patterned features (Fig. 12). The primary patterning technique is a contact-molding process which involves the use of a patterned polymeric mold to template a secondary liquid photopolymer resin layer that is subsequently UV-polymerized while in contact with the mold to give pattern transfer. The patterned polymeric mold is formed by casting a photo polymer resin on a silicon wafer, and photopolymerizing the resin to give a polymeric network mold with negative features of the original master. Polymer brushes of defined chemistry and structure are then grown from exposed embedded inimers, thus changing the size and surface chemistry of the features.

\subsection{Capillary force lithography (CFL)}

Capillary force lithography (CFL) is a simple and robust method that combines aspects of NIL and $\mu \mathrm{CP} .{ }^{104} \mathrm{CFL}$, like $\mu \mathrm{CP}$, uses an elastomeric stamp to transfer a pattern with high fidelity and in large scale onto a polymeric thin film but without the need of pressures, typical for imprint lithography. Once the polymer film is heated above its glass transition temperature, capillary forces cause the softened polymer to fill the open spaces of the elastomeric mold.

Luzinov et al. ${ }^{105}$ reported the synthesis of binary polymer brush nanopatterns on a large scale by combining CFL with SIP. CFL can achieve feature sizes on the order of $100 \mathrm{~nm}$, which opens up large-scale patterning on the nanoscale. They found the pattern can withstand subsequent polymerization conditions and stay intact during brush synthesis. The deposited PS mask on an initiator attached surface will not permit grafting

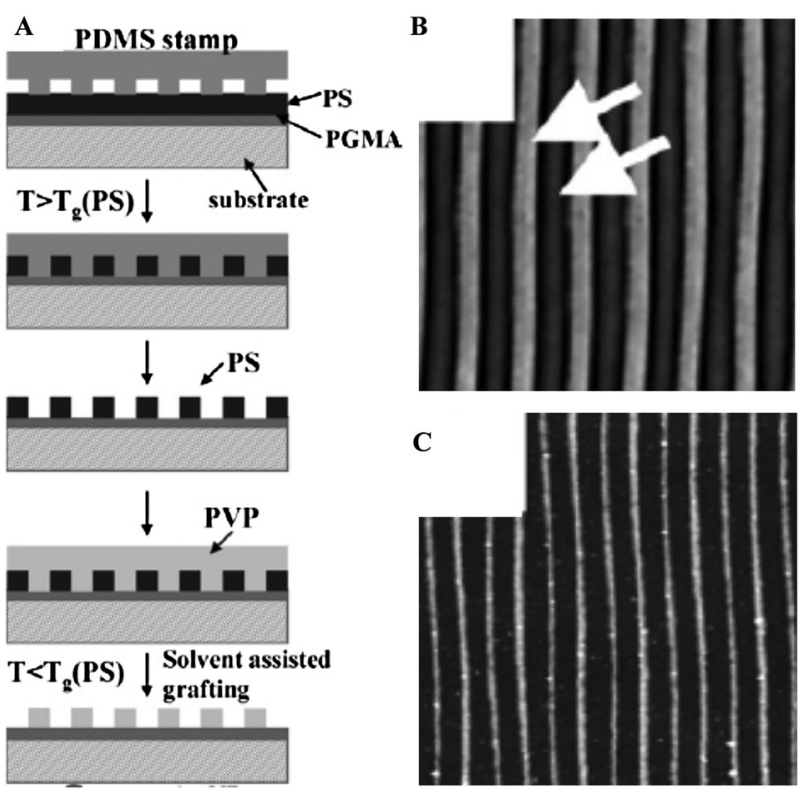

Fig. 13 (A) Scheme of patterned polymer brushes via CFL and solvent assisted grafting. (B) PS CFL on the PGMA surface (C) P2VP stripes obtained via solvent-assisted grafting ${ }^{106}$ (reproduced with permission from ref. 106, copyright 2008, Royal Society of Chemistry).

of a polymer brush in the regions underneath it, or between it and the surface. This technique was then developed by Luzinov and Zdyrko et al. ${ }^{106}$ to combine with solvent-assisted grafting approach to attach a poly-2-vinylpyridine (P2VP) onto a reactive surface forming a polymer brush by the "grafting-onto" method (Fig. 13). It can be applied to create regular patterns onto the substrate at mild reaction conditions. The uniqueness of this approach is its specifically designed grafting procedure, which allows chemical bonding of the polymer at high densities below its glass transition temperature. The patterned surfaces were obtained by protecting part of the reactive surface of epoxy functionalities poly(glycidyl methacrylate) (PGMA), followed by polymer grafting to the unprotected part of the surface.

\subsection{Colloidal lithography (CL)}

It is well known that monodisperse colloidal microspheres easily self-assemble into hexagonal close packed arrays on surfaces as a result of capillary forces arising from the evaporation of solvents. ${ }^{107}$ Such periodic arrays of microspheres were used firstly as masks in the deposition of platinum already in early 1980 by Fischer et al. ${ }^{108}$ By combination of colloidal self-assembly with nanofabrication techniques, 2D colloidal crystals have been employed as masks or templates for evaporation, deposition, and etching. These nanofabrication methods are defined as colloidal lithography (CL) and has become a simple, versatile, and cost-effective fabrication technique for a large number of researchers in the field of micro/nano fabrication. ${ }^{109}$

The powerful lithographic tool has thus shown us a possibility to structure polymer brushes. CL used for fabricating patterned polymer brushes has some advantages over the aforementioned lithographic approaches in that it employs commercially available, relatively low cost nano and micropsheres, does not require complex equipment to create micro and nanopatterned templates, and it 

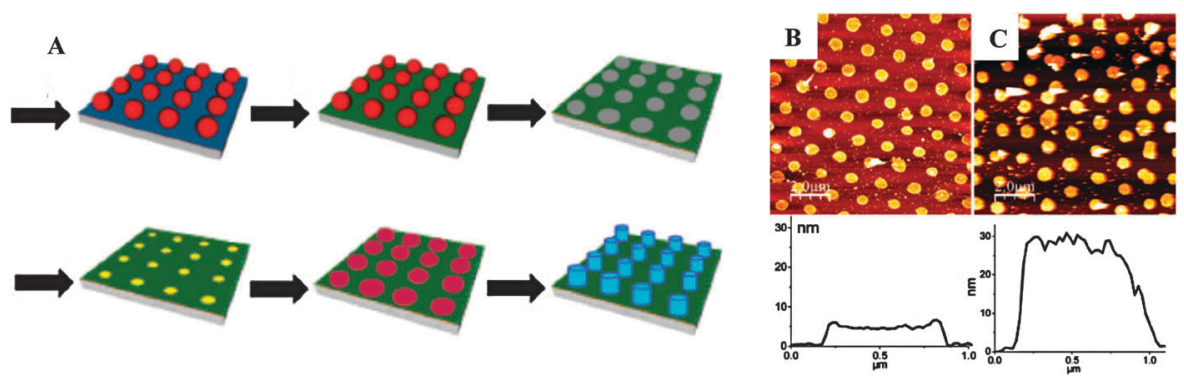

Fig. 14 (A) Scheme of patterning polymer brushes by CL/KCTP. AFM image and cross-sections of (B) micropatterned P4VP-b-PS(I) and (C) P4VP-b-PS(I)-g-P3HT brushes ${ }^{110}$ (reproduced with permission from ref. 110, copyright 2009, ACS).

allows control over polymer brush geometry by simply changing the diameter or chemical functionality of the nano or microspheres. Kiriy et al. ${ }^{110}$ reported a first example to use colloidal particle as a template for patterning polymer brushes (Fig. 14). PNIPAAM particles were prepared by a precipitation-polymerization of NIPAAM in the presence of methylenbisacrylamide crosslinker and used as a mask, and then self-assembled on silicon wafers by dip coating. Afterward, the samples were treated by octadecylsiloxane to hydrophobize the surface between the particles. The particles were then removed by ultrasonication in the acetone/water mixture and dried revealing micrometresized dots of naked silicon. Then poly(4-vinylpyridine)-blockpoly(4-iodo-styrene), P4VP-b-PS(I), in chloroform solution was adsorbed selectively onto remaining hydrophilic spots. AFM reveals the successful microstructuring of P4VP-b-PS(I) into a quasi-periodic hexagonal array of $8 \mathrm{~nm}$ in height and $\sim 1 \mu \mathrm{m}$ in diameter (Fig. 14B). Finally, the samples were activated by tetrakis(triphenylphosphine)-nickel( $(0), \mathrm{Ni}\left(\mathrm{PPh}_{3}\right)_{4}$, and poly(3-hexylthiophene) $\left(\mathrm{P}_{3} \mathrm{HT}\right)$ brushes with a height $\sim 30 \mathrm{~nm}$ was grown using Kumada catalyst-transfer polycondensation (KCTP).

Advincula et al. ${ }^{111}$ recently reported a similar approach to fabricate hole shaped patterned polymer brushes using CL and SI-ATRP. Chen et al. ${ }^{112}$ used self-assembled microsphere monolayers (SMMs) directly as stamps for $\mu \mathrm{CP}$ to fabricate patterned initiator templates that can subsequently be amplified into polymer brushes by SI-ATRP.

Another combination of SIP and colloidal particles is to modify the particles to direct self-assembly of the particles into colloidal crystals and then use the stimulus-sensitive polymer brush to control the interparticle volume. As in principle, the underlying idea is used for decades in polymer modified stationary phases for various chromatographic modes, Zharov et al. ${ }^{113}$ used colloidal films of nanoparticles modified with PNIPAAM to precisely control the interparticle volume as a function of temperature and brush layer thickness. Because of the successful developments in polymer brush synthesis, the layer dimensions that can be fine-tuned are now in the same dimension as channels or pores prepared by particle self-assembly, various other ensemble techniques e.g. using non-woven fabrics or lithography. The rapidly growing field of nanopore and nanochannel fabrication, their use in technological and biomimetic systems and their gating have been recently summarized by Jiang et al. ${ }^{114}$

\subsection{Langmuir-Blodgett lithography}

A Langmuir-Blodgett (LB) film contains one or more monolayers of an organic material at the air-water interface and can
A LB lithography using a mixed DPPC/initiator layer
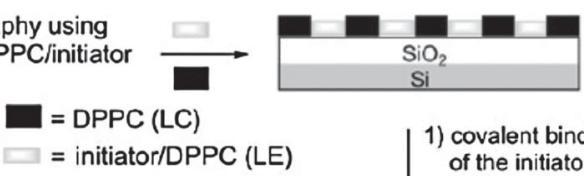

1) covalent binding of the initiator 2) removal of physi-

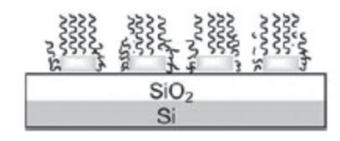

SIP sorbed DPPC
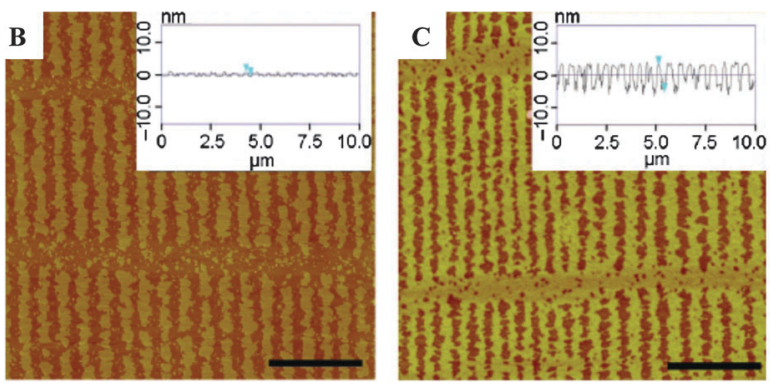

Fig. 15 (A) Formation of patterned polymer brushes by LB lithography. (B) AFM images of a sample before (B) and after (C) SIP of styrene $^{114}$ (reproduced with permission from ref. 114, copyright 2007, Wiley-VCH Verlag GmbH \& Co. KGaA).

be directly deposited onto a solid by LB transfer. Depending on the conditions, a monolayer is adsorbed homogeneously with each immersion or emersion step. Control of the dynamic parameters during the transfer results in a direct variation of the pattern features. This can be used as a patterning technique to prepare monolayer resists for nanolithography over large areas as demonstrated by Chi et al. ${ }^{115}$ Later on, Chi and Studer et al. ${ }^{116}$ used LB lithography to create stripes of enriched initiators for SI-NMP in a matrix of 1-a-dipalmitoyl-phosphatidylcholine (DPPC) (Fig. 15). The dissipative structure could be selectively amplified to lines of PS brushes as the initiator was surfacebound to the substrate by a silane function. Depending on the initiator concentration, the periodicity and stripe width can be adjusted.

\section{Conclusions and remarks}

The marriage of the top-down lithographic techniques with the bottom-up strategies of self-assembly and surface-initiated polymerization led to fantastic $2 \mathrm{D}$ and $3 \mathrm{D}$ structures of polymer brushes. Patterns on any length scale, from the centimetre down to the nanometre scale, has been demonstrated, however, 
many applications require side-by-side patterning on the microas well as the nanometre length scale and a defined periodicity. Here, not only sophisticated scanning probe based techniques or electron-beam lithography can fulfill this requirement but also more simple techniques based on self-assembly such as colloidal lithography or even microcontact printing. The challenge will be the reproducibility and degree of control. Patterning on many length scales and over large areas might also require a clever combination of different techniques that should also involve the surface-initiated polymerization to use the polymer brush itself for consecutive patterning strategies. As various lithographic techniques matured and the methods and possibilities of SIP are rapidly developing, we will soon see an increase of complexity in terms of the morphology (in 2D and 3D) as well as in the chemistry of the surface and the polymer brush. Along with numerous examples of (patterned) block copolymer brushes, first demonstrations of patterned multiple brushes have recently been shown. Besides A/B patterning, a gradual variation of brush parameters can directly be programmed onto the surface and amplified by SIP into complex 3D objects. Especially electron beam lithography or interference lithography are ideal tools to create gradual changes on the microscopic scale with nanometre precision.

As outlined, most of the approaches to fabricate patterned polymer brushes rely on patterned SAMs as they provide a defined chemical handle for consecutive SIP on many surfaces. However, this additional step of SAM deposition along with the stability issues of many SAM systems limits the use of polymer brushes for technological and biomedical applications. Moreover, many substrates are not suitable for defined SAM deposition and consecutive chemistry. A solution to this might be the use of the chemical contrast of the substrate surface itself that can be created by standard lithographic techniques with a sacrificial layer (e.g. a photoresist) or by area-selective deposition such as carbon templating (CT). Omitting SAMs of limited photo and/or thermal stability such as thiols or silanes allows for consecutive polymer analog reactions to obtain an even broader choice of polymer brushes.

As SAMs are about to leave the substrate as crosslinked nanosheets, freestanding polymer brushes, so-called polymer carpets, are following. Because both systems are very robust, can be prepared with a high aspect ratio with macroscopic lateral dimensions and nanoscopic thicknesses, these systems are promising candidates for the development of tether-free fast responding micro- or nanochemomechanical systems (M/NCMS) that will challenge existing microelectromechanical systems (MEMS).

Given the higher chemical and physical contrast along with the multiplicity of chemical functions per unit area for a polymer brush as compared to monolayer systems, potential fields for the application of patterned polymer brushes are i.e. in biomedicine for directing and control of protein adsorption and cell adhesion (non-fouling surfaces) as well as the use as (massive parallel) sensors and actuators in e.g. analytical devices for combinatorial techniques. Polymer brushes with patterned heterogeneities and periodicities of nanometre precision are ideal systems for the design of adaptive biomimetic systems responding in a unique and programmable way to their environment.

\section{Acknowledgements}

T.C. thanks the Alexander von Humboldt Foundation for support in the form of the AvH-fellowship. R.J. gratefully acknowledges constant support by the Deutsche Forschungsgemeinschaft (DFG, JO287/7-1, JO287/4-3), the IGSSE (International Graduate School of Science and Engineering, TU München) and the National Institutes of Health (NIH) (UO1 CA151806).

\section{References}

1 M. A. C. Stuart, W. T. S. Huck, J. Genzer, M. Muller, C. Ober, M. Stamm, G. B. Sukhorukov, I. Szleifer, V. V. Tsukruk, M. Urban, F. Winnik, S. Zauscher, I. Luzinov and S. Minko, Nat. Mater., 2010, 9, 101.

2 S. Minko, Polym. Rev., 2006, 46, 397.

3 T. Chen, R. Ferris, J. M. Zhang, R. Ducker and S. Zauscher, Prog. Polym. Sci., 2010, 35, 94.

4 B. Zhao and W. J. Brittain, Prog. Polym. Sci., 2000, 25, 677.

5 R. Barbey, L. Lavanant, D. Paripovic, N. Schuwer, C. Sugnaux, S. Tugulu and H. A. Klok, Chem. Rev., 2009, 109, 5437.

6 S. Edmondson, V. L. Osborne and W. T. S. Huck, Chem. Soc. Rev., 2004, 33, 14

7 Surface-Initiated Polymerization I \& II, Adv. Polym. Sci., ed. R. Jordan, Springer Verlag, 2006, vol. 197/198.

8 Polymer brushes, ed. R. C. Advincula, W. J. Brittain, K. C. Caster and J. Rühe, Wiley-VCH Verlag GmbH \& Co., 2004.

9 R. Jordan and A. Ulman, J. Am. Chem. Soc., 1998, 120, 243.

10 R. Jordan, A. Ulman, J. F. Kang, M. H. Rafailovich and J. Sokolov, J. Am. Chem. Soc., 1999, 121, 1016.

11 S. Turgman-Cohen and J. Genzer, J. Am. Chem. Soc., 2011, 133, 17567.

12 R. K. Smith, P. A. Lewis and P. S. Weiss, Prog. Surf. Sci., 2004, $75,1$.

13 Y. N. Xia and G. M. Whitesides, Angew. Chem., Int. Ed., 1998, 37, 551 .

14 A. Ulman, Chem. Rev., 1996, 96, 1533.

15 A. Ulman, An introduction to ultrathin organic films: from LangmuirBlodgett to self-assembly, Academic Press, 1991.

16 J. C. Love, L. A. Estroff, J. K. Kriebel, R. G. Nuzzo and G. M. Whitesides, Chem. Rev., 2005, 105, 1103.

17 M. Husemann, M. Morrison, D. Benoit, K. J. Frommer, C. M. Mate, W. D. Hinsberg, J. L. Hedrick and C. J. Hawker, J. Am. Chem. Soc., 2000, 122, 1844.

18 S. J. Ahn, M. Kaholek, W. K. Lee, B. LaMattina, T. H. LaBean and S. Zauscher, Adv. Mater., 2004, 16, 2141.

19 U. Schmelmer, R. Jordan, W. Geyer, W. Eck, A. Gölzhäuser, M. Grunze and A. Ulman, Angew. Chem., Int. Ed., 2003, 42, 559.

20 U. Schmelmer, A. Paul, A. Küller, M. Steenackers, A. Ulman, M. Grunze, A. Gölzhäuser and R. Jordan, Small, 2007, 3, 459.

21 M. Steenackers, A. Küller, N. Ballav, M. Zharnikov, M. Grunze and R. Jordan, Small, 2007, 3, 1764.

22 M. Kaholek, W. K. Lee, B. LaMattina, K. C. Caster and S. Zauscher, Nano Lett., 2004, 4, 373.

23 W. K. Lee, M. Patra, P. Linse and S. Zauscher, Small, 2007, 3, 63.

24 M. Husemann, D. Mecerreyes, C. J. Hawker, J. L. Hedrick, R. Shah and N. L. Abbott, Angew. Chem., Int. Ed., 1999, 38, 647.

25 F. Zhou, Z. J. Zheng, B. Yu, W. M. Liu and W. T. S. Huck, J. Am. Chem. Soc., 2006, 128, 16253.

26 J. P. Deng, W. T. Yang and B. Rånby, Macromol. Rapid Commun., 2001, 22, 535.

27 M. Steenackers, A. Küller, S. Stoycheva, M. Grunze and R. Jordan, Langmuir, 2009, 25, 2225.

28 I. Amin, M. Steenackers, N. Zhang, R. Schubel, A. Beyer, A. Gölzhäuser and R. Jordan, Small, 2011, 7, 683.

29 M. Steenackers, R. Jordan, A. Küller and M. Grunze, Adv. Mater., 2009, 21, 2921.

30 N. A. Hutter, M. Steenackers, A. Reitinger, O. A. Williams, J. A. Garrido and R. Jordan, Soft Matter, 2011, 7, 4861.

31 M. Steenackers, I. D. Sharp, K. Larsson, N. A. Hutter, M. Stutzmann and R. Jordan, Chem. Mater., 2010, 22, 272. 
32 M. Steenackers, A. M. Gigler, N. Zhang, F. Deubel, M. Seifert, L. H. Hess, C. H. Y. X. Lim, K. P. Loh, J. A. Garrido, R. Jordan, M. Stutzmann and I. D. Sharp, J. Am. Chem. Soc., 2011, 133, 10490.

33 M. Steenackers, S. Q. Lud, M. Niedermeier, P. Bruno, D. M. Gruen, P. Feulner, M. Stutzmann, J. A. Garrido and R. Jordan, J. Am. Chem. Soc., 2007, 129, 15655.

34 N. A. Hutter, A. Reitinger, N. Zhang, M. Steenackers, O. A. Williams, J. A. Garrido and R. Jordan, Phys. Chem. Chem. Phys., 2010, 12, 4360

35 T. Ito and S. Okazaki, Nature, 2000, 406, 1027.

36 O. Prucker, M. Schimmel, G. Tovar, W. Knoll and J. Rühe, $A d v$. Mater., 1998, 10, 1073.

37 O. Prucker, J. Habicht, I. J. Park and J. Rühe, Mater. Sci. Eng., C, 1999, 8-9, 291.

38 X. H. Chen, L. M. Tolbert, C. L. Henderson, D. W. Hess and J. Rühe, J. Vac. Sci. Technol., B: Microelectron. Nanometer Struct.-Process., Meas., Phenom., 2001, 19, 2013.

39 F. Zhou, L. Jiang, W. M. Liu and Q. J. Xue, Macromol. Rapid Commun., 2004, 25, 1979.

40 C. Padeste, H. H. Solak, H. P. Brack, M. Slaski, S. A. Gursel and G. G. Scherer, J. Vac. Sci. Technol., B: Microelectron. Nanometer Struct.-Process., Meas., Phenom., 2004, 22, 3191.

41 C. Schuh, S. Santer, O. Prucker and J. Rühe, Adv. Mater., 2009, 21, 4706 .

42 J. Genzer and R. R. Bhat, Langmuir, 2008, 24, 2294.

43 I. Luzinov, S. Minko and V. V. Tsukruk, Soft Matter, 2008, 4,714 .

44 T. Wu, K. Efimenko, P. Vlcek, V. Subr and J. Genzer, Macromolecules, 2003, 36, 2448.

45 L. Ionov, B. Zdyrko, A. Sidorenko, S. Minko, V. Klep, I. Luzinov and M. Stamm, Macromol. Rapid Commun., 2004, 25, 360 .

46 S. A. Campbell, The Science and Engineering of Microelectronic Fabrication, Oxford University Press, Oxford, UK, 1996, p. 203.

47 M. J. Lercel, H. G. Craighead, A. N. Parikh, K. Seshadri and D. L. Allara, Appl. Phys. Lett., 1996, 68, 1504.

48 T. Djenizian and P. Schmuki, J. Electroceram., 2006, 16, 9.

49 A. Pallandre, K. Glinel, A. M. Jonas and B. Nysten, Nano Lett., $2004,4,365$.

50 A. M. Jonas, Z. J. Hu, K. Glinel and W. T. S. Huck, Macromolecules, 2008, 41, 6859.

51 A. Rastogi, M. Y. Paik, M. Tanaka and C. K. Ober, ACS Nano, $2010,4,771$.

52 K. Slimani, L. Moine, C. Aymes-Chodur, A. Laurent, D. Labarre and N. Yagoubi, Polym. Degrad. Stab., 2009, 94, 584.

53 W. Eck, V. Stadler, W. Geyer, M. Zharnikov, A. Gölzhäuser and M. Grunze, Adv. Mater., 2000, 12, 805 .

54 S. Q. Lud, S. Neppl, G. Richter, P. Bruno, D. M. Gruen, R. Jordan, P. Feulner, M. Stutzmann and J. A. Garrido, Langmuir, 2010, 26, 15895.

55 H. L. Wang and H. R. Brown, Macromol. Rapid Commun., 2004, 25, 1095.

56 T. B. Stachowiak, F. Svec and J. M. J. Frechet, Chem. Mater., 2006, 18, 5950.

57 J. P. Deng, L. F. Wang, L. Y. Liu and W. T. Yang, Prog. Polym. Sci., 2009, 34, 156.

58 L. Faxalv, T. Ekblad, B. Liedberg and T. L. Lindahl, Acta Biomater., 2010, 6, 2599.

59 I. Amin, M. Steenackers, N. Zhang, A. Beyer, X. H. Zhang, T. Pirzer, T. Hugel, R. Jordan and A. Gölzhäuser, Small, 2010, 6, 1623 .

60 H. S. Taylor and A. A. Vernon, J. Am. Chem. Soc., 1931, 53, 2527.

61 P. White, Proc. Chem. Soc., 1961, 337.

62 A. N. Wright, Nature, 1967, 215, 953.

63 D. Braun and A. Kamprath, Angew. Makromol. Chem., 1984, $120,1$.

64 S. J. Li, C. G. Li, T. Li and J. J. Cheng, Polymer Photochemistry Principles and Applications, Fudan University Press, 1993.

65 U. Schmelmer, A. Paul, A. Küller, R. Jordan, A. Gölzhäuser, M. Grunze and A. Ulman, Macromol. Symp., 2004, 217, 223.

66 O. Prucker and J. Rühe, Macromolecules, 1998, 31, 602.

67 W. Eck, A. Küller, M. Grunze, B. Volkel and A. Gölzhäuser, Adv. Mater., 2005, 17, 2583.
68 A. Beyer, A. Godt, I. Amin, C. T. Nottbohm, C. Schmidt, J. Zhao and A. Gölzhäuser, Phys. Chem. Chem. Phys., 2008, 10, 7233.

69 J. S. Randhawa, K. E. Laflin, N. Seelam and D. H. Gracias, $A d v$. Funct. Mater., 2011, 21, 2395.

70 N. Ballav, S. Schilp and M. Zharnikov, Angew. Chem., Int. Ed., 2008, 47, 1421.

71 S. Schilp, N. Ballav and M. Zharnikov, Angew. Chem., Int. Ed., 2008, 47, 6786.

72 W. F. van Dorp and C. W. Hagen, J. Appl. Phys., 2008, 104.

73 R. D. Piner, J. Zhu, F. Xu, S. H. Hong and C. A. Mirkin, Science, 1999, 283, 661 .

74 G. Binnig and H. Rohrer, Helv. Phys. Acta, 1982, 55, 726.

75 G. Binnig, C. F. Quate and C. Gerber, Phys. Rev. Lett., 1986, 56, 930 .

76 D. Pires, J. L. Hedrick, A. De Silva, J. Frommer, B. Gotsmann, H. Wolf, M. Despont, U. Duerig and A. W. Knoll, Science, 2010, 328, 732 .

77 D. Wouters and U. S. Schubert, Angew. Chem., Int. Ed., 2004, 43, 2480.

78 F. P. Zamborini and R. M. Crooks, J. Am. Chem. Soc., 1998, 120, 9700.

79 G. Y. Liu, S. Xu and Y. L. Qian, Acc. Chem. Res., 2000, 33, 457.

80 H. Sugimura and N. Nakagiri, J. Vac. Sci. Technol., A, 1996, 14, 1223.

81 B. W. Maynor, S. F. Filocamo, M. W. Grinstaff and J. Liu, J. Am. Chem. Soc., 2002, 124, 522.

82 S. Rozhok, R. Piner and C. A. Mirkin, J. Phys. Chem. B, 2003, 107, 751.

83 X. G. Liu, S. W. Guo and C. A. Mirkin, Angew. Chem., Int. Ed., 2003, 42, 4785.

84 S. Zapotoczny, E. M. Benetti and G. J. Vancso, J. Mater. Chem., 2007, 17, 3293.

85 S. Xu and G. Y. Liu, Langmuir, 1997, 13, 127.

86 S. Xu, S. Miller, P. E. Laibinis and G. Y. Liu, Langmuir, 1999, 15,7244 .

87 X. C. Zhou, X. L. Wang, Y. D. Shen, Z. Xie and Z. J. Zheng, Angew. Chem., Int. Ed., 2011, 50, 6506.

88 M. Hirtz, M. K. Brinks, S. Miele, A. Studer, H. Fuchs and L. F. Chi, Small, $2009, \mathbf{5}, 919$.

89 A. E. Gordon, R. T. Fayfield, D. D. Litfin and T. K. Higman, J. Vac. Sci. Technol., B: Microelectron. Nanometer Struct.-Process., Meas., Phenom., 1995, 13, 2805.

90 W. K. Lee, K. C. Caster, J. Kim and S. Zauscher, Small, 2006, 2,848 .

91 A. Kumar and G. M. Whitesides, Appl. Phys. Lett., 1993, 63, 2002.

92 T. Kaufmann and B. J. Ravoo, Polym. Chem., 2010, 1, 371.

93 Y. N. Xia and G. M. Whitesides, Langmuir, 1997, 13, 2059.

94 R. B. A. Sharpe, B. J. F. Titulaer, E. Peeters, D. Burdinski, J. Huskens, H. J. W. Zandvliet, D. N. Reinhoudt and B. Poelsema, Nano Lett., 2006, 6, 1235.

95 M. Steenackers, A. Küller, N. Ballav, M. Zharnikov, M. Grunze and R. Jordan, Small, 2007, 3, 1764.

96 M. Wang, J. E. Comrie, Y. P. Bai, X. M. He, S. Y. Guo and W. T. S. Huck, Adv. Funct. Mater., 2009, 19, 2236.

97 T. Chen, J. M. Zhong, D. P. Chang, A. Carcia and S. Zauscher, Adv. Mater., 2009, 21, 1825.

98 K. Salaita, A. Amarnath, D. Maspoch, T. B. Higgins and C. A. Mirkin, J. Am. Chem. Soc., 2005, 127, 11283.

99 T. Chen, R. Jordan and S. Zauscher, Polymer, 2011, 52, 2461.

100 T. Chen, R. Jordan and S. Zauscher, Small, 2011, 7, 2148.

101 D. C. Chow and A. Chilkoti, Langmuir, 2007, 23, 11712.

102 S. Y. Chou, P. R. Krauss and P. J. Renstrom, Science, 1996, 272, 85 .

103 T. A. von Werne, D. S. Germack, E. C. Hagberg, V. V. Sheares, C. J. Hawker and K. R. Carter, J. Am. Chem. Soc., 2003, 125, 3831 .

104 K. Y. Suh, Y. S. Kim and H. H. Lee, Adv. Mater., 2001, 13, 1386.

105 Y. Liu, V. Klep and I. Luzinov, J. Am. Chem. Soc., 2006, 128, 8106.

106 B. Zdyrko, O. Hoy, M. K. Kinnan, G. Chumanov and I. Luzinov, Soft Matter, 2008, 4, 2213.

107 N. D. Denkov, O. D. Velev, P. A. Kralchevsky, I. B. Ivanov, H. Yoshimura and K. Nagayama, Nature, 1993, 361, 26. 
108 U. C. Fischer and H. P. Zingsheim, J. Vac. Sci. Technol., 1981, 19, 881. 109 J. H. Zhang, Y. F. Li, X. M. Zhang and B. Yang, Adv. Mater., 2010, 22, 4249.

110 N. Khanduyeva, V. Senkovskyy, T. Beryozkina, M. Horecha, M. Stamm, C. Uhrich, M. Riede, K. Leo and A. Kiriy, J. Am. Chem. Soc., 2009, 131, 153.

111 R. B. Pernites, E. L. Foster, M. J. L. Felipe, M. Robinson and R. C. Advincula, Adv. Mater., 2011, 23, 1287.
112 T. Chen, R. Jordan and S. Zauscher, Soft Matter, 2011, 7, 5532. 113 O. Schepelina and I. Zharov, Langmuir, 2007, 23, 12704.

114 X. Hou, W. Guo and L. Jiang, Chem. Soc. Rev., 2011, 40, 2385.

115 S. Lenhert, L. Zhang, J. Mueller, H. P. Wiesmann, G. Erker, H. Fuchs and L. F. Chi, Adv. Mater., 2004, 16, 619.

116 M. K. Brinks, M. Hirtz, L. F. Chi, H. Fuchs and A. Studer, Angew. Chem., Int. Ed., 2007, 46, 5231. 